\title{
The trion: two electrons plus one hole versus one electron plus one exciton
}

\author{
M. Combescot, O. Betbeder-Matibet and F. Dubin \\ GPS, Université Pierre et Marie Curie and Université Denis Diderot, CNRS, \\ Campus Boucicaut, 140 rue de Lourmel, 75015 Paris, France
}

\begin{abstract}
We first show that, for problems dealing with trions, it is totally hopeless to use the standard many-body description in terms of electrons and holes and its associated Feynman diagrams. We then show how, by using the description of a trion as an electron interacting with an exciton, we can obtain the trion absorption through far simpler diagrams, written with electrons and excitons. These diagrams are quite novel because, for excitons being not exact bosons, we cannot use standard procedures designed to deal with interacting true fermions or true bosons. A new many-body formalism is necessary to establish the validity of these electron-exciton diagrams and to derive their specific rules. It relies on the "commutation technique" we recently developed to treat interacting close-to-bosons. This technique generates a scattering associated to direct Coulomb processes between electrons and excitons and a dimensionless "scattering" associated to electron exchange inside the electronexciton pairs - this "scattering" being the original part of our many-body theory. It turns out that, although exchange is crucial to differentiate singlet from triplet trions, this "scattering" enters the absorption explicitly when the photocreated electron and the initial electron have the same spin - i. e., when triplet trions are the only ones created - but not when the two spins are different, although triplet trions are also created in this case. The physical reason for this rather surprising result will be given.
\end{abstract}

PACS.: 71.35.-y Excitons and related phenomena 


\section{Introduction}

While the physics of excitons and electron-hole plasma has been a subject of great interest in the 60's and 70's, the physics of trions $[1,2], i$. e., excitons bound to an electron or a hole, developed recently only: The exciton being a neutral object, its possible binding to a carrier is indeed quite weak compared to the binding of an electron to a hole. However, due to the development of nanostructure technology, it is now possible to experimentally study these trions because, all binding energies being enhanced by the reduction of dimensionality, trions, not seen in bulk samples, can appear as line well below the exciton line in the absorption spectra of doped semiconductor quantum wells (see for instance ref. $[3-9])$.

From a theoretical point of view [10-19], the study of these trions still faces major difficulties: (i) Being the eigenstates of two electrons and one hole - or two holes and one electron - in Coulomb interaction, their energies and wave functions are not analytically known, the corresponding three-body Hamiltonian having no analytical solution; (ii) because these trions are bound states, there is no way to reach them from a (finite) perturbative approach; (iii) while many-body procedures have been developed in the 60's to treat interactions between fermions (or bosons), we will show that these many-body procedures are completely inappropriate to approach the bound states resulting from the exact summation of all Coulomb processes between more than two fermions, as in the trion case.

With respect to this last point, a good idea can be to bind one electron to the hole in order for the trion to appear as a two-body system: one electron interacting with one exciton [18]. However, since the electrons are indistinguishable, the electron which is bound to the hole to make the exciton, is a priori arbitrary. Moreover - and physically linked to this arbitrariness — the exciton is not an exact boson so that standard manybody procedures, designed to treat interactions between exact fermions or exact bosons, cannot be used for this interacting electron-exciton system.

In spite of these obvious problems, the description of a trion as an electron interacting with an exciton, is physically appealing because it allows to immediately see that, as the exciton is neutral, its attraction is very weak, so that the trion binding has to be much weaker than the exciton binding. It is thus worth to find a way to cope with the difficulties 
this electron-exciton (e-X) description raises.

In order to use it, we first have to identify a quantity corresponding to the electronexciton Coulomb interaction, although, due to the composite nature of the exciton, there is no way to extract such an interaction from the Coulomb potential between individual carriers. In addition, we must find a way to take care of the electron indistinguishability when constructing the exciton. This indistinguishability somehow adds novel exchange "scatterings" to the somewhat normal electron-exciton Coulomb scatterings, with specific rules which have to be determined and which are the novel part of the diagrams corresponding to electrons interacting with excitons.

This paper in fact deals with the simplest possible problem on trions, namely the absorption of one photon in the presence of one carrier. The photon creating one electronhole (e-h) pair, we will first consider it in the framework of a two electron-one hole system, and show that the corresponding response function, written with standard electron and hole diagrams, is so complicated that there is no hope to identify and sum up the processes responsible for the trion bound states.

We will then show that this response function appears extremely simply if we bind one electron to the hole and have this exciton scattered by its interactions with the additional electron. The spin conservation of the semiconductor-photon interaction leads to differentiate absorption in which the photocreated electron and the initial electron have different spins, from absorption in which the two spins are identical. We will show that electron exchange enters the photon absorption explicitly in this last case only, $i$. e., when triplet trions are the only ones created, in spite of the fact that triplet trions can also be created when the two electrons have different spins. We will establish the rules for these electron-exciton diagrams, using our commutation technique for an exciton interacting with electrons [18]. It allows to calculate the response function to a photon field at any order in the electron-exciton interaction, while formally keeping the composite nature of the exciton, $i$. e., the fact that the exciton can be made with any of the two electrons.

This first paper allows to establish the problem of one exciton interacting with one electron on a firm basis, in order to possibly face a much harder one, namely one exciton interacting with $N$ electrons, as for the photon absorption in the presence of a Fermi sea $[11,14]$. Such an absorption has been considered long ago by Combescot and Nozières [20]. 
In this work, the spin degree of freedom of the electrons has been dropped from the first line as well as the electron-electron (e-e) repulsion. While the first simplification physically corresponds to have one kind of spin only in the problem — as for a $\sigma_{-}$photon absorbed by a quantum well having a $(+1 / 2)$ polarized Fermi sea so that $\left(S_{z}=1\right)$ triplet trions are the only ones possibly formed - , the second simplification is dramatic: Once it is made, the trion physics is irretrievably lost. Indeed, the e-e repulsion partly compensates the e-h attraction, making the trion binding energy much weaker than the exciton one, whatever the electron spins.

Let us stress that this crucial effect of the e-e interaction cannot be included through a naïve screening of the e-h attraction, as suggested by Hawrylak in his 2D extension [11] of Combescot-Nozières's work. Indeed, such a screening in the same way reduces the e-h attraction responsible for the exciton binding, so that the trion binding energy this procedure generates, is weak but equal to the exciton one, not smaller as it should. This screening procedure in fact generates one electron energy level, not two, this level being possibly occupied by two electrons if their spins are different. This procedure thus misses the whole physics of the trion, with an electron very weakly bound, compared to the other.

In order to find a trion with a binding energy much weaker than the exciton one, we must include the e-e interaction independently from any e-h process, not just through a screening of this e-h attraction. The trouble is that, within the many-body procedures at hand up to now, there is no hope to add this e-e repulsion to the set of already complicated processes which, summed up to all orders, give rise to the so called "Fermi edge singularities". In this paper, we explicitly show that the Feynman diagrams, written with electrons and holes, are already inappropriate to describe the three-body problem corresponding to just one trion. A new many-body formalism is thus highly necessary if we want to properly describe the trion absorption change with doping observed experimentally. Its presentation is in fact the underlying purpose of this work, with a particular attention paid to the interplay between the somewhat normal e-X direct Coulomb scattering and the far more subtle electron exchange inside the e-X pair.

The paper is organized as follows:

In section 2 , we briefly recall the usual procedure to calculate photon absorption and we 
try to calculate it in the case of trion formation, using the standard many-body procedure which leads to expand it through the well known electron-hole Feynman diagrams. We show that, even in the simplest case of a photon creating an electron with a spin different from the spin of the initial electron, the summation of these diagrams with all possible Coulomb processes between two electrons and one hole, is totally hopeless.

In section 3, we reconsider our description of a trion as an electron interacting with an exciton. The derivations given here of some important results on this description in terms of e-X pairs, are more direct than the somewhat pedestrian ones we gave in previous works $[17,18]$ : Once a result is mathematically established and physically understood, it becomes easy to find "straightforward" derivations and enlightening links...

In section 4 , we calculate the trion response function in terms of these interacting e-X pairs. We show that it appears as a sum of e-X ladder diagrams, with possibly one - but no more than one - electron exchange between e and X, if — and only if - the spins of the photocreated electron and the initial electron are the same. This can be surprising at first since, at each e-X scattering, the exciton can a priori be constructed with any of the two electrons, whatever their spins are.

\section{The trion as two electrons plus one hole}

A quite direct way to reach the trion is to look at the photon absorption when the semiconductor has one electron already present in the sample. Let us first briefly recall how photon absorption is usually calculated and how it appears in the case of exciton formation, since its comparison with trion is quite enlightening.

\subsection{Standard procedure to calculate photon absorption}

The linear absorption of a photon field is given by the Fermi golden rule,

$$
\frac{2 \pi}{\hbar} \sum_{F}\left|\left\langle F\left|W^{\dagger}\right| I\right\rangle\right|^{2} \delta\left(\mathbb{E}_{F}-\mathbb{E}_{I}\right)=-\frac{2}{\hbar} \operatorname{Im} S
$$

where, due to $1 /(x+i \eta)=\mathcal{P}(1 / x)-i \pi \delta(x)$, the response function $S$ reads

$$
S=\left\langle I\left|W \frac{1}{\mathbb{E}_{I}-\hat{H}+i \eta} W^{\dagger}\right| I\right\rangle
$$


as easy to show by inserting the closure relation for the $\hat{H}$ eigenstates, $|F\rangle$, in front of $W^{\dagger}$.

$\hat{H}$ is the Hamiltonian of the uncoupled matter-photon system, $\hat{H}=H+\omega_{p} \alpha^{\dagger} \alpha$, with $H$ being the matter Hamiltonian and $\alpha^{\dagger}$ the creation operator of $\left(\omega_{p}, \mathbf{Q}_{p}\right)$ photons. The coupling $W^{\dagger}$ between the photon field and the matter physically corresponds to annihilate one photon while creating one electron-hole pair, $W^{\dagger}=U^{\dagger} \alpha$. For circularly polarized photons $( \pm)$ absorbed in quantum wells, momentum conservation allows to write $U^{\dagger}$ in terms of free electrons and holes as

$$
\begin{gathered}
U_{ \pm}^{\dagger}\left(\mathbf{Q}_{p}\right)=\lambda^{*} \sum_{\mathbf{p}} a_{\mathbf{p}+\alpha_{e} \mathbf{Q}_{p}, \mp}^{\dagger} b_{-\mathbf{p}+\alpha_{h} \mathbf{Q}_{p}, \pm}^{\dagger} \\
\alpha_{e}=1-\alpha_{h}=\frac{m_{e}}{m_{e}+m_{h}}
\end{gathered}
$$

$a_{\mathbf{p}, \mp}^{\dagger}$ creates an electron with momentum $\mathbf{p}$ and spin $\mp 1 / 2$, while $b_{\mathbf{p}, \pm}^{\dagger}$ creates a hole with spin $\pm 3 / 2$. The reason for splitting $\mathbf{Q}_{p}$ between the electron and the hole as $\alpha_{e} \mathbf{Q}_{p}$ and $\alpha_{h} \mathbf{Q}_{p}$, will become apparent below.

For $N_{p}$ photons and a matter initial state $|i\rangle$ with energy $\mathbb{E}_{i}$, the initial state in eq. (2.2) reads $|I\rangle=\left|N_{p}\right\rangle \otimes|i\rangle$, the initial energy $\mathbb{E}_{I}$ being $N_{p} \omega_{p}+\mathbb{E}_{i}$. Since $\alpha\left|N_{p}\right\rangle=\sqrt{N_{p}}\left|N_{p}-1\right\rangle$, we can rewrite this response function in terms of matter quantities only, as

$$
S_{ \pm}=N_{p}\left\langle i\left|U_{ \pm}\left(\mathbf{Q}_{p}\right) \frac{1}{\omega_{p}+\mathbb{E}_{i}-H+i \eta} U_{ \pm}^{\dagger}\left(\mathbf{Q}_{p}\right)\right| i\right\rangle
$$

In eq. (2.4), $H$ acts on the photocreated electron-hole pair plus the initial carriers. Many-body effects between them follow from the identity

$$
\frac{1}{a-H}=\frac{1}{a-H_{0}}+\frac{1}{a-H} V \frac{1}{a-H_{0}},
$$

valid for $H=H_{0}+V$, which can be iterated as

$$
\frac{1}{a-H}=\frac{1}{a-H_{0}}+\frac{1}{a-H_{0}} V \frac{1}{a-H_{0}}+\cdots .
$$

In the case of semiconductors, the free part $H_{0}$ reads

$$
\begin{gathered}
H_{0}=\sum_{\mathbf{k}, s} \epsilon_{\mathbf{k}}^{(e)} a_{\mathbf{k}, s}^{\dagger} a_{\mathbf{k}, s}+\sum_{\mathbf{k}, m} \epsilon_{\mathbf{k}}^{(h)} b_{\mathbf{k}, m}^{\dagger} b_{\mathbf{k}, m} \\
\epsilon_{\mathbf{k}}^{(e, h)}=\frac{\hbar^{2} \mathbf{k}^{2}}{2 m_{e, h}}
\end{gathered}
$$


while $V$ is the Coulomb potential between carriers,

$$
\begin{array}{r}
V=\frac{1}{2} \sum_{\mathbf{q} \neq \mathbf{0}} V_{\mathbf{q}}\left[\sum_{\mathbf{k}, \mathbf{k}^{\prime}, s, s^{\prime}} a_{\mathbf{k}+\mathbf{q}, s}^{\dagger} a_{\mathbf{k}^{\prime}-\mathbf{q}, s^{\prime}}^{\dagger} a_{\mathbf{k}^{\prime}, s^{\prime}} a_{\mathbf{k}, s}+\sum_{\mathbf{k}, \mathbf{k}^{\prime}, m, m^{\prime}} b_{\mathbf{k}+\mathbf{q}, m}^{\dagger} b_{\mathbf{k}^{\prime}-\mathbf{q}, m^{\prime}}^{\dagger} b_{\mathbf{k}^{\prime}, m^{\prime}} b_{\mathbf{k}, m}\right. \\
\left.-2 \sum_{\mathbf{k}, \mathbf{k}^{\prime}, s, m^{\prime}} a_{\mathbf{k}+\mathbf{q}, s}^{\dagger} b_{\mathbf{k}^{\prime}-\mathbf{q}, m^{\prime}}^{\dagger} b_{\mathbf{k}^{\prime}, m^{\prime}} a_{\mathbf{k}, s}\right]
\end{array}
$$

with $V_{\mathbf{q}}=2 \pi e^{2} / \Omega q$ in $2 \mathrm{D}, \Omega$ being the sample volume.

\subsection{Photon absorption with exciton formation, using electron- hole diagrams}

For $\sigma_{+}$photons absorbed in an empty quantum well, the response function given in eq. (2.4) reads, due to eq. (2.3),

$$
S_{X}=N_{p}|\lambda|^{2} \sum_{\mathbf{p}^{\prime}, \mathbf{p}}\left\langle v\left|b_{-\mathbf{p}^{\prime}+\alpha_{h} \mathbf{Q}_{p},+} a_{\mathbf{p}^{\prime}+\alpha_{e} \mathbf{Q}_{p},-} \frac{1}{\omega_{p}-H+i \eta} a_{\mathbf{p}+\alpha_{e} \mathbf{Q}_{p},-}^{\dagger} b_{-\mathbf{p}+\alpha_{h} \mathbf{Q}_{p},+}^{\dagger}\right| v\right\rangle .
$$

$|v\rangle$ is the electron-hole vacuum state, its energy being chosen to be zero. $S_{X}$ can be calculated using the expansion (2.6). By noting that $\epsilon_{\mathbf{p}+\alpha_{e} \mathbf{Q}_{p}}^{(e)}+\epsilon_{-\mathbf{p}+\alpha_{h} \mathbf{Q}_{p}}^{(h)}=\epsilon_{\mathbf{p}}^{(X)}+\mathcal{E}_{\mathbf{Q}_{p}}^{(X)}$, with

$$
\epsilon_{\mathbf{p}}^{(X)}=\frac{\hbar^{2} \mathbf{p}^{2}}{2\left(m_{e}^{-1}+m_{h}^{-1}\right)^{-1}} \quad \mathcal{E}_{\mathbf{Q}}^{(X)}=\frac{\hbar^{2} \mathbf{Q}^{2}}{2\left(m_{e}+m_{h}\right)},
$$

- which is the reason for the $\left(\alpha_{e} \mathbf{Q}_{p}, \alpha_{h} \mathbf{Q}_{p}\right)$ splitting - the zero order term reduces to

$$
S_{X}^{(0)}=N_{p}|\lambda|^{2} \sum_{\mathbf{p}} \frac{1}{\omega_{p}-\epsilon_{\mathbf{p}}^{(X)}-\mathcal{E}_{\mathbf{Q}_{p}}^{(X)}+i \eta}=N_{p}|\lambda|^{2} \sum_{\mathbf{p}} G^{(e h)}\left(\omega_{p}, \mathbf{Q}_{p} ; \mathbf{p}\right)
$$

which can be used as a definition of $G^{(e h)}\left(\omega_{p}, \mathbf{Q}_{p} ; \mathbf{p}\right)$. The first order term in Coulomb interaction then appears as

$$
S_{X}^{(1)}=N_{p}|\lambda|^{2} \sum_{\mathbf{p}^{\prime}, \mathbf{p}} G^{(e h)}\left(\omega_{p}, \mathbf{Q}_{p} ; \mathbf{p}^{\prime}\right) V_{\mathbf{p}^{\prime}-\mathbf{p}} G^{(e h)}\left(\omega_{p}, \mathbf{Q}_{p} ; \mathbf{p}\right)
$$

while the second order term contains three $G^{(e h)}$ and two Coulomb potentials; and so on... This shows that the response function in the case of exciton corresponds to the well known set of electron-hole ladder diagrams [21] shown in fig. (1), since $G^{(e h)}$ is nothing but

$$
G^{(e h)}\left(\omega_{p}, \mathbf{Q}_{p} ; \mathbf{p}\right)=\int \frac{i d \omega}{2 \pi} g^{(e)}\left(\omega+\omega_{p}, \mathbf{p}+\alpha_{e} \mathbf{Q}_{p}\right) g^{(h)}\left(-\omega,-\mathbf{p}+\alpha_{h} \mathbf{Q}_{p}\right)
$$

where $g^{(e)}(\omega, \mathbf{k})=\left[\omega-\epsilon_{\mathbf{k}}^{(e)}+i \eta\right]^{-1}$ and $g^{(h)}(\omega, \mathbf{k})=\left[\omega-\epsilon_{\mathbf{k}}^{(h)}+i \eta\right]^{-1}$ are the electron propagator and hole propagator respectively, both bands being initially empty. 


\subsection{Photon absorption with trion formation, using electron-hole diagrams}

For the $X^{-}$trion to be formed, the semiconductor initial state must have one electron. If

$\mathbf{k}_{i}$ and $s_{i}$ are its momentum and spin, this initial state reads $|i\rangle=a_{\mathbf{k}_{i}, s_{i}}^{\dagger}|v\rangle$, with $\mathbb{E}_{i}=\epsilon_{\mathbf{k}_{i}}^{(e)}$. We then note that, while the photon polarization is unimportant when the semiconductor is empty, it is crucial when it already has electrons: Indeed, in a quantum well, if the spins of the photocreated electron and the initial electrons are different, the hole can only recombine with the photocreated electron, while it can recombine with any of them if their spins are identical. Consequently, the response functions differ if the electron spins are identical or not. Let us start with the simplest case.

\subsubsection{Photocreated electron with spin different from the initial one}

This happens when a $\sigma_{+}$photon is absorbed in a quantum well having a $s_{i}=+1 / 2$ electron. The zero order term of the response function appears, using eqs. $(2.3,2.4)$, as

$$
\begin{aligned}
\tilde{S}_{\neq}^{(0)}=N_{p}|\lambda|^{2} \sum_{\mathbf{p}^{\prime}, \mathbf{p}}\langle v| a_{\mathbf{k}_{i},+} b_{-\mathbf{p}^{\prime}+\alpha_{h} \mathbf{Q}_{p},+} & a_{\mathbf{p}^{\prime}+\alpha_{e} \mathbf{Q}_{p},-}\left(\frac{1}{\omega_{p}+\epsilon_{\mathbf{k}_{i}}^{(e)}-H_{0}+i \eta}\right) \\
& \times a_{\mathbf{p}+\alpha_{e} \mathbf{Q}_{p},-}^{\dagger} b_{-\mathbf{p}+\alpha_{h} \mathbf{Q}_{p},+}^{\dagger} a_{\mathbf{k}_{i},+}^{\dagger}|v\rangle .
\end{aligned}
$$

We readily find that $\tilde{S}_{\neq}^{(0)}$ reduces to the exciton zero order response function $S_{X}^{(0)}$, so that it corresponds to the diagram of fig. (1a). If we now turn to the first order term, it is given by

$$
\begin{aligned}
& \tilde{S}_{\neq}^{(1)}=N_{p}|\lambda|^{2} \sum_{\mathbf{p}^{\prime}, \mathbf{p}} G^{(e h)}\left(\omega_{p}, \mathbf{Q}_{p} ; \mathbf{p}^{\prime}\right) G^{(e h)}\left(\omega_{p}, \mathbf{Q}_{p} ; \mathbf{p}\right) \\
& \times\langle v| a_{\mathbf{k}_{i},+} b_{-\mathbf{p}^{\prime}+\alpha_{h}} \mathbf{Q}_{p},+ \\
& a_{\mathbf{p}^{\prime}+\alpha_{e} \mathbf{Q}_{p},-} V a_{\mathbf{p}+\alpha_{e} \mathbf{Q}_{p},-}^{\dagger} b_{-\mathbf{p}+\alpha_{h} \mathbf{Q}_{p},+}^{\dagger} a_{\mathbf{k}_{i},+}^{\dagger}|v\rangle
\end{aligned}
$$

where $V$ is the Coulomb potential given in eq. (2.8). As this Coulomb potential only contains $\mathbf{q} \neq \mathbf{0}$ excitations, the above matrix element differs from 0 for $\mathbf{p}^{\prime}=\mathbf{p}+\mathbf{q}$ only: This first order term is thus equal to $S_{X}^{(1)}$ and corresponds to the ladder diagram of fig. (1b).

The higher order terms are not as simple: When more than one Coulomb excitation take place, in addition to processes in which the photocreated electron is scattered several times by the hole, other processes involving the initial electron become possible: In 
addition to the exciton ladder diagrams shown in fig. (1c) at second order in $V$ and in fig. (1d) at third order, we also have the 4 diagrams of fig. (2) at second order in $V$ and the 20 diagrams of fig. (3) at third order. Note that, since the $(+1 / 2)$ electron band has one electron only, these diagrams have one conduction-hole line only, without any possible scattering, $i$. e., one electron line only going backward, from left to right.

As the $\mathrm{X}^{-}$trion corresponds to the bound state of two electrons and one hole resulting from their Coulomb interaction, it is necessary to take the Coulomb potential exactly into account $i$. e., to include it at all orders, to possibly generate the bound state poles in the response function. In view of the third order processes shown in fig. (3), it is obviously hopeless to write down the diagrams corresponding to all possible Coulomb interactions at any order in $V$ and to sum them up to get the trion.

\subsubsection{Photocreated electron with spin identical to the initial one}

The situation is worse when the photocreated electron has the spin of the initial electron, because the hole can now recombine with any of the two electrons. While the zero order term, given in eq. (2.14), with $a_{\mathbf{k}_{i},+}^{\dagger}$ replaced by $a_{\mathbf{k}_{i},-}^{\dagger}$, stays essentially unchanged, $\mathbf{p}$ being just different from $\mathbf{k}_{i}-\alpha_{e} \mathbf{Q}_{p}$, new diagrams with exchange processes between the two electrons appear at higher orders. From eq. (2.15) with $a_{\mathbf{k}_{i},+}^{\dagger}$ replaced by $a_{\mathbf{k}_{i},-}^{\dagger}$, it is easy to see that, beside $\mathbf{p}^{\prime}=\mathbf{p}+\mathbf{q}$ which gives the first order ladder diagram of fig. (1b), we can also have $\mathbf{p}=\mathbf{p}^{\prime}=\mathbf{k}_{i}-\mathbf{q}$ which gives the first order exchange diagram shown in fig. (4a). In a similar way, beside the second order direct diagrams already contained in $\tilde{S}_{\neq}^{(2)}$, we also have the six diagrams of fig. (4b) which result from exchange processes between the photocreated electron and the initial electron; and so on...

Consequently, the response function for photocreated and initial electron having the same spin, is even more complicated than the one for different spins: This leads us to conclude that the representation of a trion as two electrons plus one hole, and its associated standard electron-hole Feynman diagrams, are completely inappropriate.

We are now going to show that the description of a trion as an electron interacting with an exciton is far better. For that, let us first recall the main steps of the many-body procedure on which it is based and which allows an exact treatment of the tricky part of this description, namely the electron indistinguishability. This procedure ultimately leads 
to represent the trion through novel electron-exciton diagrams, with quite specific rules for the new "scattering" coming from pure exchange, i. e., exchange without Coulomb, not easy to guess at first.

\section{The trion as one electron plus one exciton}

By considering the trion as an electron interacting with an exciton, we tend to put the trion and the exciton on equal footing - with the hole of the exciton just replaced by an exciton. This is actually quite misleading because, due to the electron indistinguishability, the trion is definitely far more subtle than the exciton. In order to grasp the deep differences which exist between them, it appears to us useful to first recall a few well known results on exciton. In doing so, we will settle some important notations, also useful for trion.

\subsection{A few results on exciton}

\subsubsection{First quantization}

The exciton can be seen as a two-body object, made of one electron $\left(m_{e}, \mathbf{r}_{e}\right)$ and one hole $\left(m_{h}, \mathbf{r}_{h}\right)$. If we extract its center of mass, characterized by

$$
M_{X}=m_{e}+m_{h} \quad \mathbf{R}_{X}=\left(m_{e} \mathbf{r}_{e}+m_{h} \mathbf{r}_{h}\right) / M_{X}
$$

we are left with its relative motion, characterized by

$$
\mu_{X}^{-1}=m_{e}^{-1}+m_{h}^{-1} \quad \mathbf{r}=\mathbf{r}_{e}-\mathbf{r}_{h} .
$$

The exciton Hamiltonian in first quantization reads

$$
H_{X}=\frac{\mathbf{p}_{e}^{2}}{2 m_{e}}+\frac{\mathbf{p}_{h}^{2}}{2 m_{h}}-\frac{e^{2}}{\left|\mathbf{r}_{e}-\mathbf{r}_{h}\right|}=\frac{\mathbf{P}_{X}^{2}}{2 M_{X}}+h_{X}
$$

where $h_{X}=h_{X}^{(0)}-v(\mathbf{r})$ is the exciton relative motion hamiltonian composed of a freeparticle part $h_{X}^{(0)}=\mathbf{p}_{\mathbf{r}}^{2} / 2 \mu_{X}$ and a Coulomb attraction $v(\mathbf{r})=e^{2} / r$.

The eigenstates of the relative motion free part are the plane waves $|\mathbf{p}\rangle$ of energies $\epsilon_{\mathbf{p}}^{(X)}$

given in eq. (2.10): $\left(h_{X}^{(0)}-\epsilon_{\mathbf{p}}^{(X)}\right)|\mathbf{p}\rangle=0$. They are such that $\left\langle\mathbf{p}^{\prime} \mid \mathbf{p}\right\rangle=\delta_{\mathbf{p}^{\prime}, \mathbf{p}}$, their closure relation being $\sum_{\mathbf{p}}|\mathbf{p}\rangle\langle\mathbf{p}|=I$. 
The eigenstates $|\nu\rangle$ of the full relative motion Hamiltonian, $\left(h_{X}-\varepsilon_{\nu}^{(X)}\right)|\nu\rangle=0$, are made of bound and diffusive states. They are such that $\left\langle\nu^{\prime} \mid \nu\right\rangle=\delta_{\nu^{\prime}, \nu}$, their closure relation being $\sum_{\nu}|\nu\rangle\langle\nu|=I$.

In terms of $|\mathbf{p}\rangle$, these $|\nu\rangle$ states read $|\nu\rangle=\sum_{\mathbf{p}}|\mathbf{p}\rangle\langle\mathbf{p} \mid \nu\rangle$, so that the projection over $\langle\mathbf{p}|$ of the Schrödinger equation for $|\nu\rangle$ leads to

$$
\left(\epsilon_{\mathbf{p}}^{(X)}-\varepsilon_{\nu}^{(X)}\right)\langle\mathbf{p} \mid \nu\rangle-\sum_{\mathbf{p}^{\prime}}\left\langle\mathbf{p}|v(\mathbf{r})| \mathbf{p}^{\prime}\right\rangle\left\langle\mathbf{p}^{\prime} \mid \nu\right\rangle=0,
$$

the coupling being such that

$$
\left\langle\mathbf{p}|v(\mathbf{r})| \mathbf{p}^{\prime}\right\rangle=V_{\mathbf{p}^{\prime}-\mathbf{p}}
$$

where $V_{\mathbf{q}}$ is the Fourier transform of the Coulomb potential $v(\mathbf{r})$ appearing in $h_{X}$.

If we come back to the full exciton Hamiltonian, its eigenstates are the $|\nu, \mathbf{Q}\rangle$ 's with wave functions $\left\langle\mathbf{r}_{e}, \mathbf{r}_{h} \mid \nu, \mathbf{Q}\right\rangle=\langle\mathbf{r} \mid \nu\rangle\left\langle\mathbf{R}_{X} \mid \mathbf{Q}\right\rangle$, where $|\mathbf{Q}\rangle$ is the plane wave of energy $\mathcal{E}_{\mathbf{Q}}^{(X)}$ given in eq. (2.10): It is indeed easy to check that $\left(H_{X}-E_{\nu, \mathbf{Q}}^{(X)}\right)|\nu, \mathbf{Q}\rangle=0$, the total exciton energy being

$$
E_{\nu, \mathbf{Q}}^{(X)}=\varepsilon_{\nu}^{(X)}+\mathcal{E}_{\mathbf{Q}}^{(X)}
$$

\subsubsection{Second quantization}

In second quantization, the semiconductor Hamiltonian (which has the same form whatever the number of electrons and holes), is given by eqs. (2.7-8). In terms of these free electron and free hole creation operators, the creation operator for an exciton $(\nu, \mathbf{Q})$ with electron spin $s$ and hole momentum $m$ is given by

$$
B_{\nu, \mathbf{Q}, s, m}^{\dagger}=\sum_{\mathbf{p}}\langle\mathbf{p} \mid \nu\rangle a_{\mathbf{p}+\alpha_{e} \mathbf{Q}, s}^{\dagger} b_{-\mathbf{p}+\alpha_{h} \mathbf{Q}, m}^{\dagger} .
$$

Indeed, we do have $\left(H-E_{\nu, \mathbf{Q}}^{(X)}\right) B_{\nu, \mathbf{Q}, s, m}^{\dagger}|v\rangle=0$, as easy to check directly from eqs. (2.7-8), (3.4) and (3.7). Note that, with this splitting of the exciton momentum $\mathbf{Q}$ between the electron and the hole, the remaining momentum $\mathbf{p}$ is just the momentum of the exciton relative motion. It will also be useful to note that, in the same way as excitons read in terms of electron-hole pairs, electron-hole pairs can be written in terms of excitons as

$$
a_{\mathbf{p}+\alpha_{e} \mathbf{Q}, s}^{\dagger} b_{-\mathbf{p}+\alpha_{h} \mathbf{Q}, m}^{\dagger}=\sum_{\nu}\langle\nu \mid \mathbf{p}\rangle B_{\nu, \mathbf{Q}, s, m}^{\dagger},
$$

easy to check from eq. (3.7). 


\subsection{Trion in first quantization}

The trion is a three-body object (two electrons and one hole) or (two holes and one electron). To simplify the notations, we will here consider the two-electron-one-hole case only.

A first difficulty with trions, compared to excitons, arises from the spin variables. They are unimportant for excitons if we neglect "electron-hole exchange", $i$. e., valenceconduction Coulomb excitations, their energies being then degenerate with respect to $(s, m)$. On the opposite, the spin variables are crucial for trions because they differenciate their possible states through the parity of the orbital part of the wave functions with respect to the electron positions. Indeed, this parity is directly linked to the trion total electronic spin: As singlet states are odd while triplet states are even, their associate orbital wave functions must be even and odd respectively, due to the symmetry principle for the whole wave function of fermions.

Another difficulty, less obvious at first, comes from the appropriate variables to describe the trion. The center of mass $\left(M_{T}, \mathbf{R}_{T}\right)$ is surely one of these appropriate variables. For two electrons $\left(m_{e}, \mathbf{r}_{e}, \mathbf{r}_{e^{\prime}}\right)$ and one hole $\left(m_{h}, \mathbf{r}_{h}\right)$, it reads

$$
M_{T}=2 m_{e}+m_{h} \quad \mathbf{R}_{T}=\left(m_{e} \mathbf{r}_{e}+m_{e} \mathbf{r}_{e^{\prime}}+m_{h} \mathbf{r}_{h}\right) / M_{T}
$$

While for excitons, there is only one spatial variable, namely $\mathbf{r}$, which along with $\mathbf{R}_{X}$ forms a good set of variables, $i$. e., for which $\left[r_{i}, p_{j}\right]=i \hbar \delta_{i j}$, there are many ways [17] to choose the two other spatial variables which, along with $\mathbf{R}_{T}$, form a good set for trions. Among them, the convenient ones for physical understanding turn out to be $\mathbf{r}$, associated to $\mu_{X}$, defined in eq. (3.2), and $\mathbf{u}$, associated to $\mu_{T}$, defined as

$$
\mu_{T}^{-1}=m_{e}^{-1}+M_{X}^{-1} \quad \mathbf{u}=\mathbf{r}_{e^{\prime}}-\mathbf{R}_{X}
$$

$\mathbf{u}$ is the distance between $e^{\prime}$ and the center of mass of $(e, h)$, while $\mu_{T}$ is the relative motion mass of this $e^{\prime}$ electron and the $(e, h)$ exciton. Of course, due to the electron indistinguishability, variables as good as $(\mathbf{r}, \mathbf{u})$ are

$$
\mathbf{r}^{\prime}=\mathbf{r}_{e^{\prime}}-\mathbf{r}_{h}=\mathbf{u}+\alpha_{e} \mathbf{r} \quad \mathbf{u}^{\prime}=\mathbf{r}_{e}-\mathbf{R}_{X}^{\prime}=\left(1-\alpha_{e}^{2}\right) \mathbf{r}-\alpha_{e} \mathbf{u},
$$

since $\mathbf{R}_{X}^{\prime}=\left(m_{e} \mathbf{r}_{e^{\prime}}+m_{h} \mathbf{r}_{h}\right) / M_{X}$. This possible change from $(\mathbf{r}, \mathbf{u})$ to $\left(\mathbf{r}^{\prime}, \mathbf{u}^{\prime}\right)$, which corresponds to exchange the two electrons of the trion, is present all over the trion repre- 
sentation in terms of one electron plus one exciton. We will show below how to handle it in a simple way.

In terms of these variables, the trion Hamiltonian in first quantization reads

$$
\begin{aligned}
H_{T} & =\frac{\mathbf{p}_{e}^{2}}{2 m_{e}}+\frac{\mathbf{p}_{e^{\prime}}^{2}}{2 m_{e}}+\frac{\mathbf{p}_{h}^{2}}{2 m_{h}}-\frac{e^{2}}{\left|\mathbf{r}_{e}-\mathbf{r}_{h}\right|}-\frac{e^{2}}{\left|\mathbf{r}_{e^{\prime}}-\mathbf{r}_{h}\right|}+\frac{e^{2}}{\left|\mathbf{r}_{e}-\mathbf{r}_{e}^{\prime}\right|} \\
& =\frac{\mathbf{P}_{T}^{2}}{2 M_{T}}+h_{T} .
\end{aligned}
$$

Like for excitons, the relative motion Hamiltonian $h_{T}$ splits into a free part $h_{T}^{(0)}=h_{X}+$ $\mathbf{p}_{\mathbf{u}}^{2} / 2 \mu_{T}$, made of one exciton plus one effective free particle of mass $\mu_{T}$, and an interaction $w(\mathbf{r}, \mathbf{u})$ which corresponds to the Coulomb interaction between $e^{\prime}$ and $(e, h)$,

$$
w(\mathbf{r}, \mathbf{u})=\frac{e^{2}}{\left|\mathbf{r}_{e^{\prime}}-\mathbf{r}_{e}\right|}-\frac{e^{2}}{\left|\mathbf{r}_{e^{\prime}}-\mathbf{r}_{h}\right|}=\frac{e^{2}}{\left|\mathbf{u}-\alpha_{h} \mathbf{r}\right|}-\frac{e^{2}}{\left|\mathbf{u}+\alpha_{e} \mathbf{r}\right|} .
$$

The eigenstates of the relative motion free part are just the $|\nu, \mathbf{p}\rangle$ 's since we do have $h_{T}^{(0)}|\nu, \mathbf{p}\rangle=\left(\varepsilon_{\nu}^{(X)}+\epsilon_{\mathbf{p}}^{(e X)}\right)|\nu, \mathbf{p}\rangle$, with

$$
\epsilon_{\mathbf{p}}^{(e X)}=\frac{\hbar^{2} \mathbf{p}^{2}}{2 \mu_{T}} .
$$

The corresponding wave functions are $\langle\mathbf{r}, \mathbf{u} \mid \nu, \mathbf{p}\rangle=\langle\mathbf{r} \mid \nu\rangle\langle\mathbf{u} \mid \mathbf{p}\rangle$. These $|\nu, \mathbf{p}\rangle$ states are such that $\left\langle\nu^{\prime}, \mathbf{p}^{\prime} \mid \nu, \mathbf{p}\right\rangle=\delta_{\nu^{\prime}, \nu} \delta_{\mathbf{p}^{\prime}, \mathbf{p}}$, while their closure relation reads $\sum_{\nu, \mathbf{p}}|\nu, \mathbf{p}\rangle\langle\nu, \mathbf{p}|=I$.

Let us call $|\eta\rangle$ the eigenstates of the full relative motion Hamiltonian, $\left(h_{T}-\varepsilon_{\eta}^{(T)}\right)|\eta\rangle=0$. Using them, the eigenstates of the trion Hamiltonian $H_{T}$ are the $|\eta, \mathbf{K}\rangle$ 's, since we do have $\left(H_{T}-E_{\eta, \mathbf{K}}^{(T)}\right)|\eta, \mathbf{K}\rangle=0$, with

$$
E_{\eta, \mathbf{K}}^{(T)}=\varepsilon_{\eta}^{(T)}+\mathcal{E}_{\mathbf{K}}^{(T)}, \quad \mathcal{E}_{\mathbf{K}}^{(T)}=\frac{\hbar^{2} \mathbf{K}^{2}}{2 M_{T}},
$$

the corresponding wave functions being

$$
\left\langle\mathbf{r}_{e}, \mathbf{r}_{e^{\prime}}, \mathbf{r}_{h} \mid \eta, \mathbf{K}\right\rangle=\langle\mathbf{r}, \mathbf{u} \mid \eta\rangle\left\langle\mathbf{R}_{T} \mid \mathbf{K}\right\rangle
$$

As $H_{T}$, given in eq. (3.12), stays invariant under the $\left(e \leftrightarrow e^{\prime}\right)$ exchange, its eigenstates are odd or even with respect to this exchange. Since $\mathbf{R}_{T}$ is unchanged, this means that the $\langle\mathbf{r}, \mathbf{u} \mid \eta\rangle$ 's are odd or even. Let us call $\eta_{0}$, the $\eta$ indices which correspond to even functions with respect to the $\left(e \leftrightarrow e^{\prime}\right)$ exchange and $\eta_{1}$, the ones which correspond to odd functions. This parity condition thus reads, for $S=(0,1)$,

$$
\left\langle\mathbf{r}, \mathbf{u} \mid \eta_{S}\right\rangle=(-1)^{S}\left\langle\mathbf{r}^{\prime}, \mathbf{u}^{\prime} \mid \eta_{S}\right\rangle=(-1)^{S}\left\langle\mathbf{u}+\alpha_{e} \mathbf{r},\left(1-\alpha_{e}^{2}\right) \mathbf{r}-\alpha_{e} \mathbf{u} \mid \eta_{S}\right\rangle
$$


due to eq. (3.11). Within these $(\mathbf{r}, \mathbf{u})$ variables, the parity condition of the $\left|\eta_{S}\right\rangle$ 's is obviously not very appealing. We can rewrite it in a nicer form for physical understanding, by using other variables than $(\mathbf{r}, \mathbf{u})$, namely $(\nu, \mathbf{p})$. For that, we introduce the Fourier transform of $\left\langle\mathbf{r}, \mathbf{u} \mid \eta_{S}\right\rangle$ "in the exciton sense", defined as

$$
\left\langle\nu, \mathbf{p} \mid \eta_{S}\right\rangle=\int d \mathbf{r} d \mathbf{u}\langle\nu \mid \mathbf{r}\rangle\langle\mathbf{p} \mid \mathbf{u}\rangle\left\langle\mathbf{r}, \mathbf{u} \mid \eta_{S}\right\rangle
$$

We then insert eq. (3.17) into eq. (3.18), and replace $\left\langle\mathbf{r}^{\prime}, \mathbf{u}^{\prime} \mid \eta_{S}\right\rangle$ by its expression in terms of its Fourier transform, namely $\sum_{\nu^{\prime}, \mathbf{p}^{\prime}}\left\langle\mathbf{r}^{\prime} \mid \nu^{\prime}\right\rangle\left\langle\mathbf{u}^{\prime} \mid \mathbf{p}^{\prime}\right\rangle\left\langle\nu^{\prime}, \mathbf{p}^{\prime} \mid \eta_{S}\right\rangle$. Next, we express all the spatial variables in terms of $\mathbf{r}$ and $\mathbf{r}^{\prime}$, using $\mathbf{u}=\mathbf{r}^{\prime}-\alpha_{e} \mathbf{r}$ and $\mathbf{u}^{\prime}=\mathbf{r}-\alpha_{e} \mathbf{r}^{\prime}$. By noting that $\left\langle\mathbf{p} \mid \mathbf{r}^{\prime}-\alpha_{e} \mathbf{r}\right\rangle\left\langle\mathbf{r}-\alpha_{e} \mathbf{r}^{\prime} \mid \mathbf{p}^{\prime}\right\rangle$ is nothing but $\left\langle\mathbf{p}+\alpha_{e} \mathbf{p}^{\prime} \mid \mathbf{r}^{\prime}\right\rangle\left\langle\mathbf{p}^{\prime}+\alpha_{e} \mathbf{p} \mid \mathbf{r}\right\rangle$, we eventually find that the $\left|\eta_{S}\right\rangle$ 's fulfilling eq. (3.17) are such that

$$
\left\langle\nu, \mathbf{p} \mid \eta_{S}\right\rangle=(-1)^{S} \sum_{\nu^{\prime}, \mathbf{p}^{\prime}} L_{\nu \mathbf{p} ; \nu^{\prime} \mathbf{p}^{\prime}}\left\langle\nu^{\prime}, \mathbf{p}^{\prime} \mid \eta_{S}\right\rangle
$$

where $L_{\nu \mathbf{p} ; \nu^{\prime} \mathbf{p}^{\prime}}$ appears as

$$
L_{\nu \mathbf{p} ; \nu^{\prime} \mathbf{p}^{\prime}}=\left\langle\nu \mid \mathbf{p}^{\prime}+\alpha_{e} \mathbf{p}\right\rangle\left\langle\mathbf{p}+\alpha_{e} \mathbf{p}^{\prime} \mid \nu^{\prime}\right\rangle
$$

We will show below that this $L_{\nu \mathbf{p} ; \nu^{\prime} \mathbf{p}^{\prime}}$ coefficient is just the exchange "scattering" of the "commutation technique" for excitons interacting with electrons. Its link with electron exchange inside an e-X pair can however be made apparent right now, by noting that

$$
\int d \mathbf{r}_{e} d \mathbf{r}_{e^{\prime}} d \mathbf{r}_{h} \phi_{\nu^{\prime}, \mathbf{Q}^{\prime}}^{*}\left(\mathbf{r}_{e^{\prime}}, \mathbf{r}_{h}\right) f_{\mathbf{k}^{\prime}}^{*}\left(\mathbf{r}_{e}\right) \phi_{\nu, \mathbf{Q}}\left(\mathbf{r}_{e}, \mathbf{r}_{h}\right) f_{\mathbf{k}}\left(\mathbf{r}_{e^{\prime}}\right)=\delta_{\mathbf{K}^{\prime}, \mathbf{K}} L_{\nu^{\prime} \mathbf{p}^{\prime} ; \nu \mathbf{p}}
$$

where $\phi_{\nu, \mathbf{Q}}\left(\mathbf{r}_{e}, \mathbf{r}_{h}\right)=\left\langle\mathbf{r}_{e}, \mathbf{r}_{h} \mid \nu, \mathbf{Q}\right\rangle$ is the exciton wave function, $f_{\mathbf{k}}(\mathbf{r})=\langle\mathbf{r} \mid \mathbf{k}\rangle$ the free electron wave function while the $(\mathbf{Q}, \mathbf{k})$ and $(\mathbf{K}, \mathbf{p})$ are linked by

$$
\begin{gathered}
\mathbf{K}=\mathbf{Q}+\mathbf{k}, \quad \mathbf{k}=\mathbf{p}+\beta_{e} \mathbf{K} \\
\beta_{e}=1-\beta_{X}=\frac{m_{e}}{M_{T}} .
\end{gathered}
$$

Note that, in eq. (3.21), the "in" exciton $(\nu, \mathbf{Q})$ and the "out" exciton $\left(\nu^{\prime}, \mathbf{Q}^{\prime}\right)$ are made with different electrons, $\mathbf{r}_{e}$ and $\mathbf{r}_{e^{\prime}}$. The corresponding process is shown in fig. (5a). As two exchanges reduce to an identity, we do have

$$
\sum_{\nu^{\prime \prime}, \mathbf{p}^{\prime \prime}} L_{\nu^{\prime} \mathbf{p}^{\prime} ; \nu^{\prime \prime} \mathbf{p}^{\prime \prime}} L_{\nu^{\prime \prime} \mathbf{p}^{\prime \prime} ; \nu \mathbf{p}}=\delta_{\nu^{\prime}, \nu} \delta_{\mathbf{p}^{\prime}, \mathbf{p}}
$$


easy to check from eq. (3.20).

If we now come back to the $|\eta\rangle$ eigenstates of the trion relative motion Hamiltonian, the $\eta$ index is actually an $\eta_{0}$ if the trion state has a total electron spin $S=0$ and an $\eta_{1}$ if its total spin is $S=1$. This means that $\eta$ actually reads

$$
\eta=\eta_{0} \delta_{S, 0}+\eta_{1} \delta_{S, 1}
$$

where $S$ is the trion electronic spin. Let us recall that, as the trion ground state must have a symmetrical orbital wave function, its index belongs to the $\eta_{0}$ set, while its total electronic spin is $S=0$.

Since the $|\eta\rangle$ 's are eigenstates of an Hamiltonian, namely $h_{T}$, they form an orthogonal basis, so that $\left\langle\eta^{\prime} \mid \eta\right\rangle=\delta_{\eta^{\prime}, \eta}$, while their closure relation reads $\sum_{\eta}|\eta\rangle\langle\eta|=I$, the sum being taken over the $\eta_{0}$ 's and the $\eta_{1}$ 's, so that $I=I_{0}+I_{1}$, with $I_{S}=\sum_{\eta_{S}}\left|\eta_{S}\right\rangle\left\langle\eta_{S}\right|$. An interesting relation also exists for the partial sum $I_{S}$. It reads

$$
\left\langle\nu^{\prime}, \mathbf{p}^{\prime}\left|I_{S}\right| \nu, \mathbf{p}\right\rangle=\frac{1}{2}\left(\delta_{\nu^{\prime}, \nu} \delta_{\mathbf{p}^{\prime}, \mathbf{p}}+(-1)^{S} L_{\nu^{\prime} \mathbf{p}^{\prime} ; \nu \mathbf{p}}\right)
$$

and can be shown by noting that, due to eq. (3.19), $\left\langle\nu^{\prime}, \mathbf{p}^{\prime}\left|I_{S}\right| \nu, \mathbf{p}\right\rangle=(-1)^{S} \sum_{\nu^{\prime \prime}, \mathbf{p}^{\prime \prime}} L_{\nu^{\prime}} \mathbf{p}^{\prime} ; \nu^{\prime \prime} \mathbf{p}^{\prime \prime}$ $\left\langle\nu^{\prime \prime}, \mathbf{p}^{\prime \prime}\left|I_{S}\right| \nu, \mathbf{p}\right\rangle$, so that, while $\left\langle\nu^{\prime}, \mathbf{p}^{\prime}\left|I_{0}+I_{1}\right| \nu, \mathbf{p}\right\rangle=\delta_{\nu^{\prime}, \nu} \delta_{\mathbf{p}^{\prime}, \mathbf{p}}$, we do have $\left\langle\nu^{\prime}, \mathbf{p}^{\prime}\right| I_{0}-$ $I_{1}|\nu, \mathbf{p}\rangle=L_{\nu^{\prime} \mathbf{p}^{\prime} ; \nu \mathbf{p}}$. Eq. (3.25) follows from the combination of these two results.

Finally, the closure relation for the free states $|\nu, \mathbf{p}\rangle$ leads to write $|\eta\rangle$ as $\sum_{\nu, \mathbf{p}}|\nu, \mathbf{p}\rangle\langle\nu, \mathbf{p} \mid \eta\rangle$. So that the projection over $\langle\nu, \mathbf{p}|$ of the Schrödinger equation for the $|\eta\rangle$ 's gives

$$
\left(\varepsilon_{\nu}^{(X)}+\epsilon_{\mathbf{p}}^{(e X)}-\varepsilon_{\eta}^{(T)}\right)\langle\nu, \mathbf{p} \mid \eta\rangle+\sum_{\nu^{\prime}, \mathbf{p}^{\prime}}\left\langle\nu, \mathbf{p}|w(\mathbf{r}, \mathbf{u})| \nu^{\prime}, \mathbf{p}^{\prime}\right\rangle\left\langle\nu^{\prime}, \mathbf{p}^{\prime} \mid \eta\right\rangle=0
$$

the coupling being linked to the Fourier transform "in the exciton sense" of the Coulomb potential $w(\mathbf{r}, \mathbf{u})$ appearing in $h_{T}$, namely

$$
\left\langle\nu, \mathbf{p}|w(\mathbf{r}, \mathbf{u})| \nu^{\prime}, \mathbf{p}^{\prime}\right\rangle=W_{\mathbf{p}^{\prime}-\mathbf{p}}^{\nu \nu^{\prime}} \equiv\left\langle\nu\left|w_{\mathbf{p}-\mathbf{p}^{\prime}}(\mathbf{r})\right| \nu^{\prime}\right\rangle
$$

where $w_{\mathbf{q}}(\mathbf{r})=V_{\mathbf{q}}\left(e^{-i \alpha_{h} \mathbf{q} \cdot \mathbf{r}}-e^{i \alpha_{e} \mathbf{q} \cdot \mathbf{r}}\right)$ is the usual Fourier transform of $w(\mathbf{r}, \mathbf{u})$ with respect to the variable $\mathbf{u}$. Note that these results for trions are formally similar to the ones for excitons, given in eqs. (3.4-5), except for the additional exciton quantum number $\nu$.

It turns out that this coupling is just the direct Coulomb scattering $C_{\nu \mathbf{p} ; \nu^{\prime} \mathbf{p}^{\prime}}^{\operatorname{dir}}$ of the "commutation technique" for excitons interacting with electrons, we will introduce below:

$$
W_{\mathbf{p}^{\prime}-\mathbf{p}}^{\nu \nu^{\prime}} \equiv C_{\nu \mathbf{p} ; \nu^{\prime} \mathbf{p}^{\prime}}^{\operatorname{dir}}
$$


Its link to direct Coulomb processes is easy to see right now, by noting that

$$
\begin{array}{r}
\int d \mathbf{r}_{e} d \mathbf{r}_{e^{\prime}} d \mathbf{r}_{h} \phi_{\nu^{\prime}, \mathbf{Q}^{\prime}}^{*}\left(\mathbf{r}_{e}, \mathbf{r}_{h}\right) f_{\mathbf{k}^{\prime}}^{*}\left(\mathbf{r}_{e^{\prime}}\right)\left(\frac{e^{2}}{\left|\mathbf{r}_{e^{\prime}}-\mathbf{r}_{e}\right|}-\frac{e^{2}}{\left|\mathbf{r}_{e^{\prime}}-\mathbf{r}_{h}\right|}\right) \\
\phi_{\nu, \mathbf{Q}}\left(\mathbf{r}_{e}, \mathbf{r}_{h}\right) f_{\mathbf{k}}\left(\mathbf{r}_{e^{\prime}}\right) \\
=\delta_{\mathbf{K}^{\prime}, \mathbf{K}} C_{\nu^{\prime} \mathbf{p}^{\prime} ; \nu \mathbf{p}}^{\operatorname{dir}}
\end{array}
$$

where $(\mathbf{Q}, \mathbf{k} ; \mathbf{K}, \mathbf{p})$ and $\left(\mathbf{Q}^{\prime}, \mathbf{k}^{\prime} ; \mathbf{K}^{\prime}, \mathbf{p}^{\prime}\right)$ are again linked by eq. (3.22), the "in" exciton $(\nu, \mathbf{Q})$ and the "out" exciton $\left(\nu^{\prime}, \mathbf{Q}^{\prime}\right)$ being here made with the same electron $\left(\mathbf{r}_{e}\right)$. The corresponding process is shown in fig. (5b).

\subsection{Trions in second quantization}

\subsubsection{Creation operators of e-X pairs}

If we look at the expression of the exciton creation operator in terms of e-h pairs given in eq. (3.7), we see that the exciton center of mass momentum $\mathbf{Q}$ is split between the electron and the hole according to their masses, namely $\alpha_{e} \mathbf{Q}_{p}$ and $\alpha_{h} \mathbf{Q}_{p}$. In a similar way, we are led to introduce e-X pair operators with the center of mass momentum $\mathbf{K}$ split between the electron and the exciton according to their masses, namely

$$
\mathcal{T}_{\nu, \mathbf{p}, \mathbf{K} ; \sigma, s, m}^{\dagger}=a_{\mathbf{p}+\beta_{e} \mathbf{K}, \sigma}^{\dagger} B_{\nu,-\mathbf{p}+\beta_{X} \mathbf{K}, s, m}^{\dagger}
$$

with $\beta_{e}, \beta_{X}$ given in eq. (3.22).

In order to calculate the scalar product of these e-X states, it is convenient to introduce the "commutation technique" for excitons interacting with electrons. From the deviationfrom-boson operator $D_{n^{\prime} n}$ defined as $[22,23]$

$$
\left[B_{n^{\prime}}, B_{n}^{\dagger}\right]=\delta_{n^{\prime}, n}-D_{n^{\prime} n}
$$

where the $B_{n}^{\dagger}$ 's are the exciton creation operators defined in eq. (3.7) and $n$ stands for $(\nu, \mathbf{Q}, s, m)$ while $n^{\prime}$ stands for $\left(\nu^{\prime}, \mathbf{Q}^{\prime}, s^{\prime}, m^{\prime}\right)$, we find

$$
\left[D_{n^{\prime} n}, a_{\mathbf{k}, \sigma}^{\dagger}\right]=\delta_{m^{\prime}, m} \delta_{s^{\prime}, \sigma} \delta_{\mathbf{K}^{\prime}, \mathbf{K}} L_{\nu^{\prime} \mathbf{p}^{\prime} ; \nu \mathbf{p}} a_{\mathbf{k}^{\prime}, s}^{\dagger},
$$

where $(\mathbf{Q}, \mathbf{k} ; \mathbf{K}, \mathbf{p})$ and $\left(\mathbf{Q}^{\prime}, \mathbf{k}^{\prime} ; \mathbf{K}^{\prime}, \mathbf{p}^{\prime}\right)$ are linked by eq. (3.22), $L_{\nu^{\prime}} \mathbf{p}^{\prime} ; \nu \mathbf{p}$ being the parameter already appearing in eqs. (3.19-21) (see fig. (5a)).

From eqs. (3.31-32), we then readily find

$$
\left\langle v\left|\mathcal{T}_{\nu^{\prime}, \mathbf{p}^{\prime}, \mathbf{K}^{\prime} ; \sigma^{\prime}, s^{\prime}, m^{\prime}} \mathcal{T}_{\nu, \mathbf{p}, \mathbf{K} ; \sigma, s, m}^{\dagger}\right| v\right\rangle=\delta_{m^{\prime}, m} \delta_{\mathbf{K}^{\prime}, \mathbf{K}}\left(\delta_{\sigma^{\prime}, \sigma} \delta_{s^{\prime}, s} \delta_{\nu^{\prime}, \nu} \delta_{\mathbf{p}^{\prime}, \mathbf{p}}-\delta_{\sigma^{\prime}, s} \delta_{s^{\prime}, \sigma} L_{\nu^{\prime} \mathbf{p}^{\prime} ; \nu \mathbf{p}}\right)
$$


It will also be useful to note that

$$
\mathcal{T}_{\nu, \mathbf{p}, \mathbf{K} ; \sigma, s, m}^{\dagger}=-\sum_{\nu^{\prime}, \mathbf{p}^{\prime}} L_{\nu^{\prime} \mathbf{p}^{\prime} ; \nu \mathbf{p}} \mathcal{T}_{\nu^{\prime}, \mathbf{p}^{\prime}, \mathbf{K} ; s, \sigma, m}^{\dagger}
$$

which results from the two possible ways to construct a trion out of two electrons and one hole. (Note that the spins of the electron and the exciton are exchanged in the right and left hand sides of eq. (3.34)).

In the same way as exciton reads in terms of e-h pairs, trion reads in terms of e- $\mathrm{X}$ pairs. The simplest way to get this decomposition is to first find how the semiconductor Hamiltonian $H$ acts on one of this pair. For that, we again use the "commutation technique" for excitons interacting with electrons. From the Coulomb creation operator $V_{n}^{\dagger}$ defined as $[22,23]$

$$
\left[H, B_{n}^{\dagger}\right]=E_{n}^{(X)} B_{n}^{\dagger}+V_{n}^{\dagger}
$$

we find

$$
\left[V_{n}^{\dagger}, a_{\mathbf{k}, \sigma}^{\dagger}\right]=\sum_{\nu^{\prime}, \mathbf{p}^{\prime}} C_{\nu^{\prime} \mathbf{p}^{\prime} ; \nu \mathbf{p}}^{\operatorname{dir}} \mathcal{T}_{\nu^{\prime}, \mathbf{p}^{\prime}, \mathbf{K} ; \sigma, s, m}^{\dagger},
$$

where $(\mathbf{Q}, \mathbf{k} ; \mathbf{K}, \mathbf{p})$ are linked by eq. $(3.22), C_{\nu^{\prime} \mathbf{p}^{\prime} ; \nu \mathbf{p}}^{\text {dir }}$ being the quantity already appearing in eqs. (3.28-29). From eqs. (3.30), (3.35) (which implies $\left.V_{n}^{\dagger}|v\rangle=0\right)$ and (3.36), we readily get

$$
H \mathcal{T}_{\nu, \mathbf{p}, \mathbf{K} ; \sigma, s, m}^{\dagger}|v\rangle=E_{\nu \mathbf{p K}} \mathcal{T}_{\nu, \mathbf{p}, \mathbf{K} ; \sigma, s, m}^{\dagger}|v\rangle+\sum_{\nu^{\prime}, \mathbf{p}^{\prime}} C_{\nu^{\prime} \mathbf{p}^{\prime} ; \nu \mathbf{p}}^{\operatorname{dir}} \mathcal{T}_{\nu^{\prime}, \mathbf{p}^{\prime}, \mathbf{K} ; \sigma, s, m}^{\dagger}|v\rangle
$$

where $E_{\nu \mathbf{p K}}$ is the energy of the free e-X pair $(\nu, \mathbf{p}, \mathbf{K})$,

$$
E_{\nu \mathbf{p K}}=\varepsilon_{\nu}^{(X)}+\epsilon_{\mathbf{p}}^{(e X)}+\mathcal{E}_{\mathbf{K}}^{(T)}
$$

with $\epsilon_{\mathbf{p}}^{(e X)}$ and $\mathcal{E}_{\mathbf{K}}^{(T)}$ being the relative motion energy of the e-X pair and the center of mass energy of this pair, defined in eqs. (3.14-15). Note that, for $(\mathbf{Q}, \mathbf{k} ; \mathbf{K}, \mathbf{p})$ linked by eq. (3.22), we do have

$$
\epsilon_{\mathbf{k}}^{(e)}+\mathcal{E}_{\mathbf{Q}}^{(X)}=\epsilon_{\mathbf{p}}^{(e X)}+\mathcal{E}_{\mathbf{K}}^{(T)}
$$

\subsubsection{Creation operators for $\left(S_{z}= \pm 1\right)$ trions}

The trions with a spin projection $S_{z}= \pm 1$ have a total spin $S=1$, so that their relative motion index belongs to the $\eta_{1}$ set. Moreover, they have to be constructed from a $\sigma=$ $\pm 1 / 2$ electron and a $s= \pm 1 / 2$ exciton. Let us introduce the operator [18]

$$
\mathrm{T}_{\eta_{1}, \mathbf{K} ; 1, \pm 1, m}^{\dagger}=\frac{1}{\sqrt{2}} \sum_{\nu, \mathbf{p}}\left\langle\nu, \mathbf{p} \mid \eta_{1}\right\rangle \mathcal{T}_{\nu, \mathbf{p}, \mathbf{K} ; \pm 1 / 2, \pm 1 / 2, m}^{\dagger}
$$


which is similar to the exciton creation operator defined in eq. (3.7), except for the $1 / \sqrt{2}$ prefactor which is made for this operator to create a normalized two-electron-one-hole state. Indeeed, from eqs. (3.19) and (3.33), we can check that

$$
\left\langle v\left|\mathrm{~T}_{\eta_{1}, \mathbf{K} ; 1, \pm 1, m} \mathrm{~T}_{\eta_{1}, \mathbf{K} ; 1, \pm 1, m}^{\dagger}\right| v\right\rangle=\frac{1}{2} 2 \sum_{\nu, \mathbf{p}}\left|\left\langle\nu, \mathbf{p} \mid \eta_{1}\right\rangle\right|^{2}=1
$$

This $\mathrm{T}_{\nu, \mathbf{K} ; 1, \pm 1, m}^{\dagger}$ is actually the creation operator for $(S=1),\left(S_{z}= \pm 1\right)$ trions, since, due to eqs. (3.26) and (3.37), we do have

$$
H \mathrm{~T}_{\eta_{1}, \mathbf{K} ; 1, \pm 1, m}^{\dagger}|v\rangle=E_{\eta_{1}, \mathbf{K}}^{(T)} \mathrm{T}_{\eta_{1}, \mathbf{K} ; 1, \pm 1, m}^{\dagger}|v\rangle
$$

with

$$
E_{\eta_{S}, \mathbf{K}}^{(T)}=\varepsilon_{\eta_{S}}^{(T)}+\mathcal{E}_{\mathbf{K}}^{(T)}
$$

\subsubsection{Creation operators for $\left(S_{z}=0\right)$ trions}

$\left(S_{z}=0\right)$ trions have a total spin $S$ either equal to 0 or to 1 , so that their relative motion indices can be either an $\eta_{0}$ or an $\eta_{1}$. Moreover, they can be built either from a $(\sigma=1 / 2)$ electron and a $(s=-1 / 2)$ exciton, or the reverse. However, as the two corresponding e-X operators are linked by the relation (3.34), we are led to introduce just the first type of e-X pairs [18],

$$
\mathrm{T}_{\eta_{S}, \mathbf{K} ; S, 0, m}^{\dagger}=\sum_{\nu, \mathbf{p}}\left\langle\nu, \mathbf{p} \mid \eta_{S}\right\rangle \mathcal{T}_{\nu, \mathbf{p}, \mathbf{K} ;+1 / 2,-1 / 2, m}^{\dagger}
$$

since from eqs. (3.19) and (3.34), this operator also reads

$$
\mathrm{T}_{\eta_{S}, \mathbf{K} ; S, 0, m}^{\dagger}=-(-1)^{S} \sum_{\nu, \mathbf{p}}\left\langle\nu, \mathbf{p} \mid \eta_{S}\right\rangle \mathcal{T}_{\nu, \mathbf{p}, \mathbf{K} ;-1 / 2,+1 / 2, m}^{\dagger}
$$

which makes clear the fact that such a $\left(S_{z}=0\right)$ trion can be built either from a $(\sigma=1 / 2)$ electron and a $(s=-1 / 2)$ exciton or from a $(\sigma=-1 / 2)$ electron and a $(s=1 / 2)$ exciton.

Using again eqs. (3.26) and (3.37), it is straightforward to check that this $\mathrm{T}_{\eta_{S}, \mathbf{K} ; S, 0, m}^{\dagger}$ is indeed a trion creation operator, since we do have

$$
H \mathrm{~T}_{\eta_{S}, \mathbf{K} ; S, 0, m}^{\dagger}|v\rangle=E_{\eta_{S}, \mathbf{K}}^{(T)} \mathrm{T}_{\eta_{S}, \mathbf{K} ; S, 0, m}^{\dagger}|v\rangle
$$

while, from eqs. (3.19) and (3.33), one can check that it indeed creates a normalized $\left(S_{z}=0\right)$ trion state. 
Let us end by noting that the trion states defined above form an orthogonal basis, since we do have

$$
\left\langle v\left|\mathrm{~T}_{\eta_{S^{\prime}}^{\prime}, \mathbf{K}^{\prime} ; S^{\prime}, S_{z}^{\prime}, m^{\prime}} \mathrm{T}_{\eta_{S}, \mathbf{K} ; S, S_{z}, m}^{\dagger}\right| v\right\rangle=\delta_{S^{\prime}, S} \delta_{S_{z}^{\prime}, S_{z}} \delta_{m^{\prime}, m} \delta_{\eta_{S^{\prime}}^{\prime}, \eta_{S}} \delta_{\mathbf{K}^{\prime}, \mathbf{K}}
$$

while their closure relation reads

$$
1=\sum_{S, S_{z}, m, \eta_{S}, \mathbf{K}} \mathrm{T}_{\eta_{S}, \mathbf{K} ; S, S_{z}, m}^{\dagger}|v\rangle\langle v| \mathrm{T}_{\eta_{S}, \mathbf{K} ; S, S_{z}, m}
$$

Finally, it is easy to check that, in the same way as trions can be written in terms of e-X pairs according to eqs. (3.40) and (3.44), e-X pairs can be written in terms of trions, according to

$$
\begin{gathered}
\mathcal{T}_{\nu, \mathbf{p}, \mathbf{K} ; \pm 1 / 2 ; \pm 1 / 2, m}^{\dagger}=\sqrt{2} \sum_{\eta_{1}}\left\langle\eta_{1} \mid \nu, \mathbf{p}\right\rangle \mathrm{T}_{\eta_{1}, \mathbf{K} ; 1, \pm 1, m}^{\dagger}, \\
\mathcal{T}_{\nu, \mathbf{p}, \mathbf{K} ;+1 / 2 ;-1 / 2, m}^{\dagger}=\sum_{S, \eta_{S}}\left\langle\eta_{S} \mid \nu, \mathbf{p}\right\rangle \mathrm{T}_{\eta_{S}, \mathbf{K} ; S, 0, m}^{\dagger}, \\
\mathcal{T}_{\nu, \mathbf{p}, \mathbf{K} ;-1 / 2 ;+1 / 2, m}^{\dagger}=-\sum_{S, \eta_{S}}(-1)^{S}\left\langle\eta_{S} \mid \nu, \mathbf{p}\right\rangle \mathrm{T}_{\eta_{S}, \mathbf{K} ; S, 0, m}^{\dagger} .
\end{gathered}
$$

Although somewhat more complicated due to the importance of spins for trions, these equations are the analogues of eq. (3.8) relating free e-h pairs to excitons.

\subsection{Many-body effects between electrons and excitons}

In usual many-body problems, the Hamiltonian splits as $H=H_{0}+V$, so that the manybody effects result from eq. (2.5) and its iteration (2.6). In the case of many-body effects with excitons, such a separation of the Hamiltonian is not possible, due to the composite nature of the exciton. Attempts have been made to produce a potential $V_{X X}$ between excitons by bosonizing them. However, quite recently, we have shown that these procedures, although rather sophisticated, fail to give the correct answer to physical quantities such as the exciton lifetime and the exciton-exciton scattering rate [25], whatever the X-X scattering used in $V_{X X}$ is. We have also shown that they fail to give the correct nonlinear susceptibilities [26], because they miss purely Pauli many-body effects, $i$. e., scattering

processes which exist in the absence of any Coulomb interaction. This is why we will not here use the bosonization procedures to describe the interaction of one electron with one exciton. 
It is actually possible to handle many-body effects with exact excitons properly, by noting that eq. (3.35) leads to [24]

$$
\frac{1}{a-H} B_{n}^{\dagger}=B_{n}^{\dagger} \frac{1}{a-H-E_{n}^{(X)}}+\frac{1}{a-H} V_{n}^{\dagger} \frac{1}{a-H-E_{n}^{(X)}} .
$$

The above equation, which is the equivalent of eq. (2.5) for usual many-body effects, is the key equation for many-body effects involving excitons. It cannot be iterated as simply as eq. (2.6). It is however possible to generate such an iteration by having eq. (3.52) acting on excitons or on electrons and by using either $\left[V_{n}^{\dagger}, B_{n}^{\dagger}\right]$ given in eq. (3) of ref. [22] for many-body effects between excitons, or $\left[V_{n}^{\dagger}, a_{\mathbf{k}, \sigma}^{\dagger}\right]$, given in eq. (3.36), for many-body effects between excitons and electrons.

\subsection{1 $(a-H)^{-1}$ acting on e-X pairs}

Equations (3.36) and (3.52) give $(a-H)^{-1}$ acting on one e-X pair as [18]

$$
\frac{1}{a-H} \mathcal{T}_{\nu, \mathbf{p}, \mathbf{K} ; \sigma, s, m}^{\dagger}|v\rangle=\left[\mathcal{T}_{\nu, \mathbf{p}, \mathbf{K} ; \sigma, s, m}^{\dagger}|v\rangle+\sum_{\nu^{\prime}, \mathbf{p}^{\prime}} \frac{1}{a-H} \mathcal{T}_{\nu^{\prime}, \mathbf{p}^{\prime}, \mathbf{K} ; \sigma, s, m}^{\dagger}|v\rangle C_{\nu^{\prime} \mathbf{p}^{\prime} ; \nu \mathbf{p}}^{\mathrm{dir}}\right] \frac{1}{a-E_{\nu \mathbf{p K}}},
$$

where $E_{\nu \mathbf{p K}}$ is the free e-X pair energy given in eq. (3.38).

If we now iterate eq. (3.53), we find

$$
\frac{1}{a-H} \mathcal{T}_{\nu, \mathbf{p}, \mathbf{K} ; \sigma, s, m}^{\dagger}|v\rangle=\sum_{\nu^{\prime}, \mathbf{p}^{\prime}} A_{\nu^{\prime} \mathbf{p}^{\prime} ; \nu \mathbf{p}}(a, \mathbf{K}) \mathcal{T}_{\nu^{\prime}, \mathbf{p}^{\prime}, \mathbf{K} ; \sigma, s, m}^{\dagger}|v\rangle
$$

where the prefactor $A_{\nu^{\prime} \mathbf{p}^{\prime} ; \nu \mathbf{p}}(a, \mathbf{K})$ expands on the e-X direct Coulomb scatterings only, through

$$
\begin{aligned}
A_{\nu^{\prime} \mathbf{p}^{\prime} ; \nu \mathbf{p}}(a, \mathbf{K})=\frac{\delta_{\nu^{\prime}, \nu} \delta_{\mathbf{p}^{\prime}, \mathbf{p}}}{a-E_{\nu \mathbf{p K}}} & +\frac{C_{\nu^{\prime} \mathbf{p}^{\prime} ; \nu \mathbf{p}}^{\operatorname{dir}}}{\left(a-E_{\nu^{\prime} \mathbf{p}^{\prime} \mathbf{K}}\right)\left(a-E_{\nu \mathbf{p K}}\right)} \\
& +\sum_{\nu_{1}, \mathbf{p}_{1}} \frac{C_{\nu^{\prime} \mathbf{p}^{\prime} ; \nu_{1} \mathbf{p}_{1}}^{\operatorname{dir}} C_{\nu_{1} \mathbf{p}_{1} ; \nu \mathbf{p}}^{\operatorname{dir}}}{\left(a-E_{\nu^{\prime} \mathbf{p}^{\prime} \mathbf{K}}\right)\left(a-E_{\nu_{1} \mathbf{p}_{1} \mathbf{K}}\right)\left(a-E_{\nu \mathbf{p K}}\right)}+\cdots
\end{aligned}
$$

It corresponds to the ladder processes between electron and exciton shown in fig. (6).

Just as the summation of the e-h ladder processes producing the exciton reads in terms of exciton energies and wave functions, the summation of these e-X ladder processes producing the trion should read in terms of trion energies and wave functions. Let us now show it. 


\subsubsection{Sum of e-X ladder processes}

If we take the scalar product of $\langle v| \mathcal{T}_{\nu^{\prime \prime}}, \mathbf{p}^{\prime \prime}, \mathbf{K} ;+1 / 2,-1 / 2, m$ with eq. (3.54) taken for $\sigma=-s=$ $1 / 2$, we find from equation (3.33)

$$
\left\langle v\left|\mathcal{T}_{\nu^{\prime \prime}, \mathbf{p}^{\prime \prime}, \mathbf{K} ;+1 / 2,-1 / 2, m} \frac{1}{a-H} \mathcal{T}_{\nu, \mathbf{p}, \mathbf{K} ;+1 / 2,-1 / 2, m}^{\dagger}\right| v\right\rangle=A_{\nu^{\prime \prime} \mathbf{p}^{\prime \prime} ; \nu \mathbf{p}}(a, \mathbf{K})
$$

If we now insert the trion closure relation (3.48) in the LHS of the above equation, we get, using eqs. (3.42) and (3.46),

$$
\begin{aligned}
& A_{\nu^{\prime \prime} \mathbf{p}^{\prime \prime} ; \nu \mathbf{p}}(a, \mathbf{K})=\sum_{S^{\prime}, S_{Z}^{\prime}, m^{\prime}, \eta_{S^{\prime}}^{\prime}, \mathbf{K}^{\prime}} \\
& \frac{\left\langle v\left|\mathcal{T}_{\nu^{\prime \prime}, \mathbf{p}^{\prime \prime}, \mathbf{K} ;+1 / 2,-1 / 2, m} \mathrm{~T}_{\eta_{S^{\prime}}^{\prime}, \mathbf{K}^{\prime} ; S^{\prime}, S_{z}^{\prime} ; m^{\prime}}^{\dagger}\right| v\right\rangle\left\langle v\left|\mathrm{~T}_{\eta_{S^{\prime}}^{\prime}, \mathbf{K}^{\prime} ; S^{\prime}, S_{z}^{\prime} ; m^{\prime}} \mathcal{T}_{\nu, \mathbf{p}, \mathbf{K} ;+1 / 2,-1 / 2, m}^{\dagger}\right| v\right\rangle}{a-E_{\eta_{S^{\prime}}^{\prime}, \mathbf{K}^{\prime}}^{(T)}} .
\end{aligned}
$$

From the expansion of e-X pairs in terms of trions given in eq. (3.50), we immediately find, since trions form an orthogonal basis,

$$
\begin{gathered}
A_{\nu^{\prime \prime} \mathbf{p}^{\prime \prime} ; \nu \mathbf{p}}(a, \mathbf{K})=\sum_{S=0,1} A_{\nu^{\prime \prime} \mathbf{p}^{\prime \prime} ; \nu \mathbf{p}}^{(S)}(a, \mathbf{K}), \\
A_{\nu^{\prime \prime} \mathbf{p}^{\prime \prime} ; \nu \mathbf{p}}^{(S)}(a, \mathbf{K})=\sum_{\eta_{S}} \frac{\left\langle\nu^{\prime \prime}, \mathbf{p}^{\prime \prime} \mid \eta_{S}\right\rangle\left\langle\eta_{S} \mid \nu, \mathbf{p}\right\rangle}{a-E_{\eta_{S}, \mathbf{K}}^{(T)}} .
\end{gathered}
$$

We see that $A_{\nu^{\prime \prime} \mathbf{p}^{\prime \prime} ; \nu \mathbf{p}}(a, \mathbf{K})$ contains contributions from both, singlet trions $(S=0)$ and triplet trions $(S=1)$.

For physical understanding, it can be of interest to note that, if we set $a^{\prime}=a-\mathcal{E}_{\mathbf{K}}^{(T)}$, the compact expression of $A_{\nu^{\prime \prime}} \mathbf{p}^{\prime \prime} ; \nu \mathbf{p}(a, \mathbf{K})$ in terms of trions, given in eqs. (3.58-59), reads

$$
A_{\nu^{\prime \prime} \mathbf{p}^{\prime \prime} ; \nu \mathbf{p}}(a, \mathbf{K})=\hat{A}_{\nu^{\prime \prime} \mathbf{p}^{\prime \prime} ; \nu \mathbf{p}}\left(a^{\prime}\right)=\left\langle\nu^{\prime \prime}, \mathbf{p}^{\prime \prime}\left|\frac{1}{a^{\prime}-h_{\mathrm{T}}}\right| \nu, \mathbf{p}\right\rangle
$$

while its expansion in e-X Coulomb scatterings given in eq. (3.55) just corresponds to

$$
\hat{A}_{\nu^{\prime \prime} \mathbf{p}^{\prime \prime} ; \nu \mathbf{p}}\left(a^{\prime}\right)=\left\langle\nu^{\prime \prime}, \mathbf{p}^{\prime \prime}\left|\frac{1}{a^{\prime}-h_{T}^{(0)}}\right| \nu, \mathbf{p}\right\rangle+\left\langle\nu^{\prime \prime}, \mathbf{p}^{\prime \prime}\left|\frac{1}{a^{\prime}-h_{T}^{(0)}} w(\mathbf{r}, \mathbf{u}) \frac{1}{a^{\prime}-h_{T}^{(0)}}\right| \nu, \mathbf{p}\right\rangle+\cdots
$$

due to the link between $C_{\nu^{\prime} \mathbf{p}^{\prime} ; \nu \mathbf{p}}^{\mathrm{dir}}$ and $w(\mathbf{r}, \mathbf{u})$ given in eqs. (3.27-28). It is then obvious that eq. (3.61) just follows from eq. (3.60), since for $h_{T}=h_{T}^{(0)}+w(\mathbf{r}, \mathbf{u})$, we do have

$$
\frac{1}{a^{\prime}-h_{T}}=\frac{1}{a^{\prime}-h_{T}^{(0)}}+\frac{1}{a^{\prime}-h_{T}^{(0)}} w(\mathbf{r}, \mathbf{u}) \frac{1}{a^{\prime}-h_{T}^{(0)}}+\cdots .
$$


Let us stress that the summation of e-X ladder processes has first been established from a quite formal procedure designed to treat electrons interacting with excitons. It can a priori be used in any other problem dealing with electrons and excitons, not just in the case of one electron plus one exciton, $i$. e., one trion. It is however clear that we can also use it in this simple problem too, for which a formulation in first quantization through the trion Hamiltonian $H_{T}\left(\mathbf{R}_{T}, \mathbf{r}, \mathbf{u}\right)$ is simple enough to be of practical use. This first quantization formulation of the one-trion problem actually provides enlightening foreshortenings to some results on e-X pairs. In more complicated situations however, second quantization along with the formal definitions of the direct Coulomb and exchange scatterings between electrons and excitons it allows, are the only possible way to treat problems dealing with one hole and more than two electrons. The two aspects of the same results are however of interest for the understanding of the trion physics.

\section{Photon absorption using electron-exciton diagrams}

In section 2, we have derived the photon absorption using e-h diagrams in the case of exciton formation and in the case of trion formation. We have shown that these standard Feynman diagrams are in fact totally inappropriate for trions. In section 3 , we have derived all the tools necessary to propose a new diagrammatic procedure for photon absorption in terms of excitons, while taking into account the fact that the exciton can also be made with any of the electrons present in the sample, through the exchange "scatterings" generated by the "commutation technique".

This new formulation of photon absorption is in fact quite natural: Indeed, the semiconductor-photon interaction, given in eq. (2.3) in terms of free electrons and free holes, can also be written in terms of excitons. From eq. (3.8) and the fact that $\sqrt{\Omega}\langle\mathbf{p}| \mathbf{r}=$ $\mathbf{0}\rangle=1$, we readily get

$$
\begin{gathered}
U_{ \pm}^{\dagger}\left(\mathbf{Q}_{p}\right)=\sum_{\nu} \lambda_{\nu}^{*} B_{\nu, \mathbf{Q}_{p}, \mp, \pm}^{\dagger} \\
\lambda_{\nu}^{*}=\lambda^{*} \sqrt{\Omega}\langle\nu \mid \mathbf{r}=\mathbf{0}\rangle .
\end{gathered}
$$

This just corresponds to the well known enhancement factor of the coupling to exciton compared to free pairs, as $|\langle\nu \mid \mathbf{r}=\mathbf{0}\rangle|^{2}$ is of the order of the inverse exciton volume: With respect to this enhanced coupling already, the exciton representation appears to us 
somewhat better.

\subsection{Photon absorption with exciton formation}

From the response function given in eq. (2.4) in the case of a semiconductor with no carrier, irradiated with $\sigma_{+}$photons, we get

$$
S_{X}=N_{p} \sum_{\nu^{\prime}, \nu} \lambda_{\nu^{\prime}}\left\langle v\left|B_{\nu^{\prime}, \mathbf{Q},-,+} \frac{1}{\omega_{p}-H+i \eta} B_{\nu, \mathbf{Q}_{p},-,+}^{\dagger}\right| v\right\rangle \lambda_{\nu}^{*} .
$$

This readily gives

$$
S_{X}=N_{p} \sum_{\nu} \lambda_{\nu} G^{(X)}\left(\omega_{p}, \mathbf{Q}_{p} ; \nu\right) \lambda_{\nu}^{*}
$$

in which we have set

$$
G^{(X)}(\omega, \mathbf{Q} ; \nu)=\frac{1}{\omega-E_{\nu, \mathbf{Q}}^{(X)}+i \eta} .
$$

$G^{(X)}(\omega, \mathbf{Q} ; \nu)$ can be seen as an exciton propagator; so that we are led to represent $S_{X}$ by the exciton diagram of fig. (7a), which is already far simpler than the set of electron-hole ladder diagrams of fig. (1).

\subsection{Photon absorption with trion formation}

From eqs. (2.4) and (4.1), the response function for an initial state already having one electron with momentum $\mathbf{k}_{i}$ and spin $s_{i}$ and an absorbed photon $\sigma_{ \pm}$, reads

$$
S_{ \pm, s_{i}}=N_{p} \sum_{\nu^{\prime}, \nu} \lambda_{\nu^{\prime}}\left\langle v\left|a_{\mathbf{k}_{i}, s_{i}} B_{\nu^{\prime}, \mathbf{Q}_{p}, \mp, \pm}\left(\frac{1}{\omega_{p}+\epsilon_{\mathbf{k}_{i}}^{(e)}-H+i \eta}\right) B_{\nu, \mathbf{Q}_{p}, \mp, \pm}^{\dagger} a_{\mathbf{k}_{i}, s_{i}}^{\dagger}\right| v\right\rangle \lambda_{\nu}^{*} .
$$

If we introduce the appropriate momenta $\left(\mathbf{p}_{i}, \mathbf{K}_{i}\right)$ of the e-X pair made of the initial electron and the photocreated virtual exciton, defined by

$$
\mathbf{K}_{i}=\mathbf{k}_{i}+\mathbf{Q}_{p}, \quad \mathbf{k}_{i}=\mathbf{p}_{i}+\beta_{e} \mathbf{K}_{i}
$$

we can rewrite $S_{ \pm, s_{i}}$ as

$$
S_{ \pm, s_{i}}=N_{p} \sum_{\nu^{\prime}, \nu} \lambda_{\nu^{\prime}}\left\langle v\left|\mathcal{T}_{\nu^{\prime}, \mathbf{p}_{i}, \mathbf{K}_{i} ; s_{i}, \mp, \pm}\left(\frac{1}{\omega_{p}+\epsilon_{\mathbf{k}_{i}}^{(e)}-H+i \eta}\right) \mathcal{T}_{\nu, \mathbf{p}_{i}, \mathbf{K}_{i} ; s_{i}, \mp, \pm}^{\dagger}\right| v\right\rangle \lambda_{\nu}^{*} .
$$

So that, by using eq. (3.54), which gives $(a-H)^{-1}$ acting on one e-X pair, we find

$$
S_{ \pm, s_{i}}=N_{p} \sum_{\nu^{\prime}, \nu} \sum_{\nu_{1}, \mathbf{p}_{1}} \lambda_{\nu^{\prime}}\left\langle v\left|\mathcal{T}_{\nu^{\prime}, \mathbf{p}_{i}, \mathbf{K}_{i} ; s_{i}, \mp, \pm} \mathcal{T}_{\nu_{1}, \mathbf{p}_{1}, \mathbf{K}_{i} ; s_{i}, \mp, \pm}^{\dagger}\right| v\right\rangle A_{\nu_{1} \mathbf{p}_{1} ; \nu \mathbf{p}_{i}}\left(a_{i}, \mathbf{K}_{i}\right) \lambda_{\nu}^{*}
$$


with $a_{i}=\omega_{p}+\epsilon_{\mathbf{k}_{i}}^{(e)}+i \eta$. In order to go further, we note that the scalar product of e-X states, given by eq. (3.33), depends if the photocreated electron and the initial electron have the same spin or not.

\subsection{Photocreated electron with spin different from the initial one}

When the spins are different, the scalar product of e-X states appearing in eq. (4.9) differs from zero for $\nu_{1}=\nu^{\prime}$ and $\mathbf{p}_{1}=\mathbf{p}_{i}$ only, so that we simply have

$$
S_{\neq}=N_{p} \sum_{\nu^{\prime}, \nu} \lambda_{\nu^{\prime}} A_{\nu^{\prime} \mathbf{p}_{i} ; \nu \mathbf{p}_{i}}\left(a_{i}, \mathbf{K}_{i}\right) \lambda_{\nu}^{*}
$$

Due to eq. (3.58), the response function contains contributions from singlet trions $(S=0)$ and triplet trions $(S=1)$ as expected, since with two different electron spins, we can construct the two types of trions. By using the expansion of $A_{\nu^{\prime} \mathbf{p}^{\prime} ; \nu \mathbf{p}}(a, \mathbf{K})$ in direct Coulomb scatterings given in eq. (3.55), we can expand $S_{\neq}$as

$$
S_{\neq}=\sum_{n=0}^{+\infty} S_{\neq}^{(n)}
$$

where $S_{\neq}^{(n)}$ has $n$ electron-exciton scatterings $C_{\nu_{1} \mathbf{p}_{1} ; \nu_{2} \mathbf{p}_{2}}^{\text {dir }}$.

\subsubsection{Zero order term in $\mathrm{e}-\mathrm{X}$ interactions}

The term without any e-X scattering is given by

$$
S_{\neq}^{(0)}=N_{p} \sum_{\nu} \frac{\left|\lambda_{\nu}\right|^{2}}{a_{i}-E_{\nu \mathbf{p}_{i} \mathbf{K}_{i}}}=N_{p} \sum_{\nu} \lambda_{\nu} G^{(X)}\left(\omega_{p}, \mathbf{Q}_{p} ; \nu\right) \lambda_{\nu}^{*},
$$

and corresponds to the diagram of fig. (7a). Before going further, let us note that we can rewrite this zero order term in a compact form, without any explicit reference to exciton states, as

$$
S_{\neq}^{(0)}=N_{p}|\lambda|^{2} \Omega\left\langle\mathbf{r}=\mathbf{0}\left|\frac{1}{\omega_{p}-\left(h_{X}+\mathcal{E}_{\mathbf{Q}_{p}}^{(X)}\right)+i \eta}\right| \mathbf{r}=\mathbf{0}\right\rangle,
$$

where $h_{X}$ is the exciton relative motion Hamiltonian, $\left(h_{X}-\epsilon_{\nu}^{(X)}\right)|\nu\rangle=0$. 


\subsubsection{First order term}

The first order term in e-X scattering is zero since direct Coulomb processes impose nonzero momentum transfers, so that $C_{\nu^{\prime} \mathbf{p}^{\prime} ; \nu \mathbf{p}}^{\mathrm{dir}}=0$ for $\mathbf{p}^{\prime}=\mathbf{p}$ (see eqs. (3.27-28)). This has to be contrasted with e-h diagrams for which a first order term exists. Let us however stress that this e-h diagram first order term is just a part of the ladder processes giving rise to the exciton propagator, so that it is in fact already included in the zero order exciton diagram of fig. (7a).

\subsubsection{Second order term}

Using eqs. (3.28) and (3.55), the second order term in e-X scatterings reads

$$
S_{\neq}^{(2)}=N_{p} \sum_{\nu^{\prime}, \nu} \lambda_{\nu^{\prime}} G^{(X)}\left(\omega_{p}, \mathbf{Q}_{p} ; \nu^{\prime}\right)\left[\sum_{\nu_{1}, \mathbf{q}_{1}} \frac{W_{-\mathbf{q}_{1}}^{\nu^{\prime} \nu_{1}} W_{\mathbf{q}_{1}}^{\nu_{1} \nu}}{\Delta_{\nu_{1}, \mathbf{q}_{1}}}\right] G^{(X)}\left(\omega_{p}, \mathbf{Q}_{p} ; \nu\right) \lambda_{\nu}^{*}
$$

in which we have set

$$
\Delta_{\nu, \mathbf{q}}=\omega_{p}-\left(E_{\nu, \mathbf{Q}_{p}+\mathbf{q}}^{(X)}+\epsilon_{\mathbf{k}_{i}-\mathbf{q}}^{(e)}-\epsilon_{\mathbf{k}_{i}}^{(e)}\right)+i \eta
$$

It is easy to check that the bracket of the above equation can also be written as

$$
\sum_{\nu_{1}, \mathbf{q}_{1}} \int \frac{i d \omega_{1}}{2 \pi} W_{-\mathbf{q}_{1}}^{\nu^{\prime} \nu_{1}} B\left(\omega_{1}, \mathbf{q}_{1}\right) G_{X}\left(\omega_{p}+\omega_{1}, \mathbf{Q}_{p}+\mathbf{q}_{1} ; \nu_{1}\right) W_{\mathbf{q}_{1}}^{\nu_{1} \nu},
$$

where $B\left(\omega_{1}, \mathbf{q}_{1}\right)$ is the standard bubble for the excitation of one e-h pair in the "Fermi sea", here reduced to the $\left(\mathbf{k}_{i}, s_{i}\right)$ electron, namely

$$
B\left(\omega_{1}, \mathbf{q}_{1}\right)=(-1) \sum_{\mathbf{k}} \int \frac{i d \omega}{2 \pi} g_{i}^{(e)}(\omega, \mathbf{k}) g_{i}^{(e)}\left(\omega-\omega_{1}, \mathbf{k}-\mathbf{q}_{1}\right) .
$$

where $g_{i}^{(e)}(\omega, \mathbf{k})=\left(\omega-\epsilon_{\mathbf{k}}^{(e)}+i \eta S_{\mathbf{k}, \mathbf{k}_{i}}\right)^{-1}$, with $S_{\mathbf{k}, \mathbf{k}_{i}}=+1$ if $\mathbf{k} \neq \mathbf{k}_{i}$ and $S_{\mathbf{k}, \mathbf{k}_{i}}=-1$ if $\mathbf{k}=\mathbf{k}_{i}$. Consequently, this second order term just corresponds to the diagram of fig. (7b), with the e-X direct scattering vertex given by eq. (3.27).

It is also possible to rewrite this second order term in a compact form, without any reference to exciton states, as

$$
\begin{array}{r}
S_{\neq}^{(2)}=N_{p}|\lambda|^{2} \Omega\langle\mathbf{r}=\mathbf{0}| \frac{1}{\omega_{p}-\left(h_{X}+\mathcal{E}_{\mathbf{Q}_{p}}^{(X)}\right)+i \eta} \\
\times\left[\sum_{\mathbf{q}_{1}} w_{\mathbf{q}_{1}}(\mathbf{r}) \frac{1}{\omega_{p}-\left(h_{X}+\mathcal{E}_{\mathbf{Q}_{p}+\mathbf{q}_{1}}^{(X)}+\epsilon_{\mathbf{k}_{i}-\mathbf{q}_{1}}^{(e)}-\epsilon_{\mathbf{k}_{i}}^{(e)}\right)+i \eta} w_{-\mathbf{q}_{1}}(\mathbf{r})\right] \\
\times \frac{1}{\omega_{p}-\left(h_{X}+\mathcal{E}_{\mathbf{Q}_{p}}^{(X)}\right)+i \eta}|\mathbf{r}=\mathbf{0}\rangle .
\end{array}
$$


This unique diagram $(7 \mathrm{~b})$ has to be contrasted to the six diagrams corresponding to the second order term in $V_{\mathbf{q}}$ of the standard e-h many-body procedure (the second order ladder diagram of fig. (1c) plus the 5 diagrams of fig. (2)). Let us again note that the e-X and e-h many-body procedures are not strictly equivalent: Diagram (7b) contains terms in $V_{\mathbf{q}}^{(n)}$ with $n>2$, not included in fig. (2). They are somehow "hidden" in the exciton

propagator $G^{(X)}$ which in fact includes all ladder processes between the photocreated electron and the hole.

\subsubsection{Third order term}

In the same way, the third order term in e-X interactions reads

$$
\begin{gathered}
S_{\neq}^{(3)}=N_{p} \sum_{\nu^{\prime}, \nu} \lambda_{\nu^{\prime}} G^{(X)}\left(\omega_{p}, \mathbf{Q}_{p} ; \nu^{\prime}\right) \\
\times\left[\sum_{\nu_{1}, \nu_{2} ; \mathbf{q}_{1}, \mathbf{q}_{2}} \frac{W_{-\mathbf{q}_{2}}^{\nu^{\prime} \nu_{2}} W_{\mathbf{q}_{2}-\mathbf{q}_{1}}^{\nu_{2} \nu_{1}} W_{\mathbf{q}_{1}}^{\nu_{1} \nu}}{\Delta_{\nu_{2}, \mathbf{q}_{2}} \Delta_{\nu_{1}, \mathbf{q}_{1}}}\right] G_{X}\left(\omega_{p}, \mathbf{Q}_{p} ; \nu\right) \lambda_{\nu}^{*} .
\end{gathered}
$$

Using the exciton-photon vertex, the e-X direct scattering and the exciton propagator defined above, as well as the $(\omega, \mathbf{q})$ conservations standard for diagrams, it is easy to show that this third order term just corresponds to the diagram of fig. (7c).

This unique diagram has again to be contrasted with the twenty one diagrams of the e-h Feynman diagram procedure at third order in Coulomb interaction (the third order ladder diagram of fig. (1d) plus the 20 diagrams of fig. (3)): This again shows that our e-X diagrams are far more convenient than the usual e-h Feynman diagrams, for this simple problem on trions already. In more complicated problems, they should be even more convenient. This is why, by using them, we can have some hope to calculate quantities which may appear as impossible to obtain from the usual e-h many-body procedure.

Before going further, we can note that this third order term also takes the compact form 


$$
\begin{array}{r}
S_{\neq}^{(3)}=N_{p}|\lambda|^{2} \Omega\langle\mathbf{r}=\mathbf{0}| \frac{1}{\omega_{p}-\left(h_{X}+\mathcal{E}_{\mathbf{Q}_{p}}^{(X)}\right)+i \eta} \\
\times\left[\sum_{\mathbf{q}_{1}, \mathbf{q}_{2}} w_{\mathbf{q}_{2}}(\mathbf{r}) \frac{1}{\omega_{p}-\left(h_{X}+\mathcal{E}_{\mathbf{Q}_{p}+\mathbf{q}_{2}}^{(X)}+\epsilon_{\mathbf{k}_{i}-\mathbf{q}_{2}}^{(e)}-\epsilon_{\mathbf{k}_{i}}^{(e)}\right)+i \eta}\right. \\
\left.\times w_{\mathbf{q}_{1}-\mathbf{q}_{2}}(\mathbf{r}) \frac{1}{\omega_{p}-\left(h_{X}+\mathcal{E}_{\mathbf{Q}_{p}+\mathbf{q}_{1}}^{(X)}+\epsilon_{\mathbf{k}_{i}-\mathbf{q}_{1}}^{(e)}-\epsilon_{\mathbf{k}_{i}}^{(e)}\right)+i \eta} w_{-\mathbf{q}_{1}}(\mathbf{r})\right] \\
\times \frac{1}{\omega_{p}-\left(h_{X}+\mathcal{E}_{\mathbf{Q}_{p}}^{(X)}\right)+i \eta}|\mathbf{r}=\mathbf{0}\rangle .
\end{array}
$$

\subsubsection{Higher order terms}

While we did not even dare to draw the e-h Feynman diagrams at fourth order in Coulomb interaction, the simplicity of the e-X diagram procedure may lead us to think that the fourth order terms in e-X interactions should be given by the ladder diagram of fig. (7d). And similarly at higher orders. The correct result is somewhat more subtle as we now show.

The standard diagrammatic procedure with electron propagators, that we partly use here, for example in the bubble $B\left(\omega_{1}, \mathbf{q}_{1}\right)$ of eq. (4.17), is actually designed to describe e-h excitations of the Fermi sea, $i$. e., processes in which $\mathbf{k} \neq \mathbf{k}-\mathbf{q}_{n}$. While at first and second order in e-X Coulomb processes, this is automatically fulfilled due to the $\mathbf{q} \neq \mathbf{0}$ constraint on $W_{\nu^{\prime} \nu}(\mathbf{q})$ scatterings, this is no more imposed at higher orders. Indeed, while in the fourth order term of $A_{\nu^{\prime}} \mathbf{p}_{i} ; \nu \mathbf{p}_{i}\left(a_{i}, \mathbf{K}_{i}\right)$, the first and last scatterings still impose $\mathbf{q}_{1} \neq \mathbf{0}$ and $\mathbf{q}_{3} \neq \mathbf{0}$, the intermediate scatterings simply impose $\left(\mathbf{q}_{2}-\mathbf{q}_{1}\right) \neq \mathbf{0}$ and $\left(\mathbf{q}_{3}-\mathbf{q}_{2}\right) \neq \mathbf{0}$ so that we can possibly have $\mathbf{q}_{2}=\mathbf{0}$. However, the precise calculation of the ladder diagram of fig. (7d) confirms that this diagram only has excited Fermi sea e-h pairs $\left(\mathbf{k}, \mathbf{k}-\mathbf{q}_{n}\right)$ with $\mathbf{q}_{n} \neq \mathbf{0}$ : The $\mathbf{q}_{2}=0$ ones are missing. Since the expansion of $A_{\nu^{\prime} \mathbf{p}_{i} ; \nu \mathbf{p}_{i}}\left(a_{i}, \mathbf{K}_{i}\right)$ does contain all possible scatterings of the photocreated exciton, $i$. e., the $\mathbf{q}_{n}=\mathbf{0}$ scatterings too, we must add the diagram of fig. (7e) to the one of fig. (7d), in order to have all the fourth order terms of $S_{\neq}$. We can then be tempted to separate this additional diagram (and the similar ones at higher orders) from the set of ladder diagrams with 0, 1, 2 scatterings between the photocreated exciton and the electron, in which the exciton is always in a state $\mathbf{Q} \neq \mathbf{Q}_{p}$. As explained in more details below, this idea, which can appear physically 
appealing, turns out to be a bad one.

To conclude, we can say that, when the photocreated electron and the initial electron have different spins, the response function $S_{\neq}$corresponds to all possible diagrams between one electron and one exciton, with the following characteristics:

(i) These diagrams have one exciton line only, going from right to left, since there is one deep hole only: the photocreated one.

(ii) The photocreated (virtual) exciton suffers various scatterings, without any electron exchange with the initial electron, because, for two electrons having different spins, the deep hole can only recombine with the photocreated electron.

(iii) These diagrams have a unique conduction-hole line, going from left to right: Since the initial state has one electron $\left(\mathbf{k}_{i}, s_{i}\right)$ only, the corresponding initial "Fermi sea" can have one hole only, with a well defined momentum, namely $\mathbf{k}_{i}$, so that this hole cannot scatter. With such an essentially empty "Fermi sea", the conduction electron $\left(s_{i}\right)$ has, on the opposite, plenty of sites to scatter.

\subsubsection{Summation of e-X ladder diagrams}

In the case of a photocreated electron with spin different from the initial one, the trion absorption response function $S_{\neq}$thus corresponds to the set of e-X ladder diagrams shown in fig. (7). They contain direct Coulomb processes only, the exciton being always made with the same electron.

These diagrams can be summed up in terms of the trion relative motion energies and wave functions. Using eqs. (3.58-59), we find

$$
A_{\nu^{\prime} \mathbf{p}_{i} ; \nu \mathbf{p}_{i}}\left(a_{i}, \mathbf{K}_{i}\right)=\sum_{S} \sum_{\eta_{S}} \frac{\left\langle\nu^{\prime}, \mathbf{p}_{i} \mid \eta_{S}\right\rangle\left\langle\eta_{S} \mid \nu, \mathbf{p}_{i}\right\rangle}{a_{i}-E_{\eta_{S}, \mathbf{K}_{i}}^{(T)}}
$$

where $\mathbf{p}_{i}$ is the relative motion momentum of the e-X pair made of the initial electron and the photocreated exciton defined in eq. (4.7). According to eqs. (4.2,4.10), this leads to

$$
S_{\neq}=N_{p}|\lambda|^{2} \Omega \sum_{S} \sum_{\eta_{S}} \frac{\left|\left\langle\mathbf{r}=\mathbf{0}, \mathbf{p}_{i} \mid \eta_{S}\right\rangle\right|^{2}}{\omega_{p}-\left(E_{\eta_{S}, \mathbf{K}_{i}}^{(T)}-\epsilon_{\mathbf{k}_{i}}^{(e)}\right)+i \eta},
$$

in agreement with our previous work on trion absorption [19].

By using the trion relative motion Hamiltonian $h_{T}$, which is such that 


$$
\begin{aligned}
& \left(h_{T}-\varepsilon_{\eta_{S}}^{(T)}\right)\left|\eta_{S}\right\rangle=0 \text {, eq. (4.22) also reads } \\
& \qquad S_{\neq}=N_{p}|\lambda|^{2} \Omega\left\langle\mathbf{r}=\mathbf{0}, \mathbf{p}_{i}\left|\frac{1}{\omega_{p}-\left(h_{T}+\mathcal{E}_{\mathbf{K}_{i}}^{(T)}-\epsilon_{\mathbf{k}_{i}}^{(e)}\right)+i \eta}\right| \mathbf{r}=\mathbf{0}, \mathbf{p}_{i}\right\rangle,
\end{aligned}
$$

From the above result, it is easy to recover the compact expressions of the various terms of $S_{\neq}$given in eqs. $(4.13,4.18,4.20)$. Indeed, since $h_{T}=h_{T}^{(0)}+w(\mathbf{r}, \mathbf{u})$, we get, from eq. (4.23) and the expansion of $1 /\left(a^{\prime}-h_{T}\right)$ given in eq. (3.62),

$$
S_{\neq}=N_{p}|\lambda|^{2} \Omega\left\langle\mathbf{r}=\mathbf{0}, \mathbf{p}_{i}\left|\frac{1}{a_{i}^{\prime}-h_{T}^{((0)}}+\frac{1}{a_{i}^{\prime}-h_{T}^{(0)}} w(\mathbf{r}, \mathbf{u}) \frac{1}{a_{i}^{\prime}-h_{T}^{(0)}}+\cdots\right| \mathbf{r}=\mathbf{0}, \mathbf{p}_{i}\right\rangle,
$$

where $a_{i}^{\prime}=\omega_{p}+\epsilon_{\mathbf{k}_{i}}^{(e)}-\mathcal{E}_{\mathbf{K}_{i}}^{(T)}+i \eta$. We then insert the closure relation for free e-X states $|\nu, \mathbf{p}\rangle$ in front of each $1 /\left(a_{i}^{\prime}-h_{T}^{(0)}\right)$ factor. The $S_{\neq}$zero order term is proportional to

$$
\left\langle\mathbf{r}=\mathbf{0}, \mathbf{p}_{i}\left|\frac{1}{a_{i}^{\prime}-h_{T}^{(0)}}\right| \mathbf{r}=\mathbf{0}, \mathbf{p}_{i}\right\rangle=\left\langle\mathbf{r}=\mathbf{0}\left|\frac{1}{a_{i}^{\prime}-h_{X}-\epsilon_{\mathbf{p}_{i}}^{(e X)}}\right| \mathbf{r}=\mathbf{0}\right\rangle,
$$

which is nothing but the expectation value of $1 /\left(\omega_{p}-h_{X}-\mathcal{E}_{\mathbf{Q}_{p}}^{(X)}+i \eta\right)$ in the $|\mathbf{r}=\mathbf{0}\rangle$ state, in agreement with eq. (4.13).

The first order term of $S_{\neq}$is proportional to

$$
\sum_{\nu^{\prime}, \mathbf{p}^{\prime}, \nu, \mathbf{p}}\left\langle\mathbf{r}=\mathbf{0}, \mathbf{p}_{i}\left|\frac{1}{a_{i}^{\prime}-h_{T}^{(0)}}\right| \nu^{\prime}, \mathbf{p}^{\prime}\right\rangle\left\langle\nu^{\prime}, \mathbf{p}^{\prime}|w(\mathbf{r}, \mathbf{u})| \nu, \mathbf{p}\right\rangle\left\langle\nu, \mathbf{p}\left|\frac{1}{a_{i}^{\prime}-h_{T}^{(0)}}\right| \mathbf{r}=\mathbf{0}, \mathbf{p}_{i}\right\rangle,
$$

which imposes $\mathbf{p}^{\prime}=\mathbf{p}_{i}=\mathbf{p}$. It is thus equal to zero, since Coulomb scatterings are restricted to non-zero momentum transfers only.

In a similar way, the second order term is proportional to

$$
\begin{gathered}
\sum_{\nu^{\prime}, \mathbf{p}^{\prime}, \nu, \mathbf{p}, \nu_{1}, \mathbf{p}_{1}, \nu_{2}, \mathbf{p}_{2}}\left\langle\mathbf{r}=\mathbf{0}, \mathbf{p}_{i}\left|\frac{1}{a_{i}^{\prime}-h_{T}^{(0)}}\right| \nu^{\prime}, \mathbf{p}^{\prime}\right\rangle\left\langle\nu^{\prime}, \mathbf{p}^{\prime}|w(\mathbf{r}, \mathbf{u})| \nu_{2}, \mathbf{p}_{2}\right\rangle \\
\times\left\langle\nu_{2}, \mathbf{p}_{2}\left|\frac{1}{a_{i}^{\prime}-h_{T}^{(0)}}\right| \nu_{1}, \mathbf{p}_{1}\right\rangle\left\langle\nu_{1}, \mathbf{p}_{1}|w(\mathbf{r}, \mathbf{u})| \nu, \mathbf{p}\right\rangle\left\langle\nu, \mathbf{p}\left|\frac{1}{a_{i}^{\prime}-h_{T}^{(0)}}\right| \mathbf{r}=\mathbf{0}, \mathbf{p}_{i}\right\rangle,
\end{gathered}
$$

which imposes $\mathbf{p}^{\prime}=\mathbf{p}_{i}=\mathbf{p}$ and $\mathbf{p}_{2}=\mathbf{p}_{1}$. By writing $\mathbf{p}_{1}=\mathbf{p}_{i}-\mathbf{q}_{1}$, it is straightforward to check that eq. (4.27) indeed leads to eq. (4.18).

And so on, for the higher order terms.

\subsubsection{An inappropriate separation}

Let us end this part by explaining why it is inappropriate to treat separately the diagrams in which the scattered excitons have a center of mass momentum $\mathbf{Q}_{p}+\mathbf{q}_{n}$ equal to its initial value $\mathbf{Q}_{p}$. 
With such a separation, we would be led to write the response function $S_{\neq}$as in fig. (8a), where the cross represents all topologically connected e-X ladder diagrams shown in fig. (8b), i. e., diagrams in which all intermediate excitons have their center of mass momentum $\mathbf{Q}_{p}+\mathbf{q}_{n}$ different from $\mathbf{Q}_{p}$. Fig. (8a) would lead to write $S_{\neq}$as

$$
S_{\neq}=N_{p} \sum_{\nu^{\prime}, \nu} \lambda_{\nu^{\prime}}\left[\delta_{\nu^{\prime}, \nu} G_{X}\left(\omega_{p}, \mathbf{Q}_{p} ; \nu\right)+G_{X}\left(\omega_{p}, \mathbf{Q}_{p} ; \nu^{\prime}\right) \Gamma_{\nu^{\prime} \nu} G_{X}\left(\omega_{p}, \mathbf{Q}_{p} ; \nu\right)+\cdots\right] \lambda_{\nu}^{*}
$$

From the results obtained in the above section, it is easy to show that the exciton scattering $\Gamma_{\nu^{\prime} \nu}$ associated to the cross has the form $\left\langle\nu^{\prime}\left|T\left(\omega_{p}, \mathbf{Q}_{p}, \mathbf{k}_{i}\right)\right| \nu\right\rangle$; so that all these diagrams can be summed up as

$$
\begin{aligned}
\left\langle\nu^{\prime}\right| \frac{1}{\omega_{p}-h_{X}-\mathcal{E}_{\mathbf{Q}_{p}}^{(X)}+i \eta}+\frac{1}{\omega_{p}-h_{X}-\mathcal{E}_{\mathbf{Q}_{p}}^{(X)}+i \eta} T\left(\omega_{p}, \mathbf{Q}_{p}, \mathbf{k}_{i}\right) \\
\quad \times \frac{1}{\omega_{p}-h_{X}-\mathcal{E}_{\mathbf{Q}_{p}}^{(X)}+i \eta}+\cdots|\nu\rangle=\left\langle\nu^{\prime}\left|\frac{1}{\omega_{p}-\tilde{h}_{X}-\mathcal{E}_{\mathbf{Q}_{p}}^{(X)}+i \eta}\right| \nu\right\rangle
\end{aligned}
$$

where $\tilde{h}_{X}$, equal to $h_{X}+T\left(\omega_{p}, \mathbf{Q}_{p}, \mathbf{k}_{i}\right)$, appears as the Hamiltonian of the photocreated exciton dressed by its possible scatterings with the initial electron.

While physically appealing at first, this concept turns out to be technically useless: Indeed, the sum of e-X ladder processes can be summed up easily in terms of singlet and triplet trions when - and only when - all $\mathbf{q}_{n}$ 's are included. On the opposite, the sum of ladder processes restricted to $\mathbf{q}_{n} \neq \mathbf{0}$ as the ones appearing in $T\left(\omega_{p}, \mathbf{Q}_{p}, \mathbf{k}_{i}\right)$ is not known. Consequently, although physically nice at first, eq. (4.29) which would lead to write the response function as

$$
S_{\neq}=N_{p}|\lambda|^{2} \Omega\left\langle\mathbf{r}=\mathbf{0}\left|\frac{1}{\omega_{p}-\tilde{h}_{X}-\mathcal{E}_{\mathbf{Q}_{p}}^{(X)}+i \eta}\right| \mathbf{r}=\mathbf{0}\right\rangle,
$$

is of no use. If we now take a mathematical point of view, we hardly see how the one-body operator $\tilde{h}_{X}$ can be transformed into a two-body operator, for the $|\nu, \mathbf{p}\rangle$ (or $\left.|\mathbf{r}, \mathbf{p}\rangle\right)$ states to appear in the response function as they should, in view of eq. (4.23). By comparing eqs. (4.23) and (4.30), we note that the matrix elements between $|\mathbf{r}=\mathbf{0}\rangle$ states of $\left(\omega_{p}-\right.$ $\left.\tilde{h}_{X}-\mathcal{E}_{\mathbf{Q}_{p}}^{(X)}+i \eta\right)^{-1}$ and $\left\langle\mathbf{p}_{i}\left|\left[\omega_{p}-\left(h_{T}+\mathcal{E}_{\mathbf{K}_{i}}^{(T)}-\epsilon_{\mathbf{k}_{i}}^{(e)}\right)+i \eta\right]^{-1}\right| \mathbf{p}_{i}\right\rangle$ must be equal. This however does not tell anything about the operators themselves. 


\subsubsection{Conclusion}

The response function, in the case of a photocreated electron with spin different from the initial one, is very similar to the response function of an exciton: It just contains e-X ladder processes. It is however of importance to stress that, while, in the exciton case, there is no way to cut these ladder diagrams into pieces, in the trion case, the exciton - which plays the role of the hole in the exciton ladder processes - can possibly return into a $\left(\nu_{n}, \mathbf{Q}_{p}\right)$ state after a set of scatterings. This makes the corresponding diagram topologically separable. These two types of diagrams have however to be included in order to find all terms appearing in the response function (see figs. (7d) and (7e)). Another important point is the fact that no additional diagram resulting from possible electron exchanges exists, although, at any stage, the exciton can a priori be made either with the photocreated electron or with the initial electron. This is basically due to the fact that, in a quantum well, when the electron spins are different, the hole can only recombine with the photocreated electron; so that the number of exchanges which can take place before recombination, has to be even. Since two exchanges reduce to an identity according to eq. (3.23), two exchanges are basically the same as no exchange at all.

A last comment: For most people, "trion" in fact means "ground state trion". Such trion corresponds to singlet state, $i$. e., state in which the two electrons have different spins, so that it corresponds to the e-X ladder diagrams described in this paragraph. The real challenging difficulty with trions seen as interacting e-X pairs, in fact arises when the two electrons have the same spin, $i$. e., when the hole can recombine with any of the two electrons, so that electron exchanges have to explicitly enter the problem. Let us end this work by considering these triplet trions, although they are not the interesting ones in most experiments.

\subsection{Photocreated electron with spin identical to the initial one}

The consequences of the possible electron exchanges in forming the exciton can appear quite difficult to handle at first. This is probably why trions have not been treated as a set of interacting e-X pairs up to now. Let us show how our commutation technique allows to take care of this electron exchange in a simple and transparent way.

From a rapid look at eq. (4.22), we see that the response function $S_{\neq}$for different 
electron spins reads in terms of trion states $\left|\eta_{S}\right\rangle$ with both $S=0$ and $S=1$. This is reasonable since, with $|+-\rangle$ electrons, we can form singlet and triplet trions. On the opposite, if the two electrons have the same spin, $|++\rangle$ for example, we can only form $(S=1)$ trions, so that only $(S=1)$ trions should enter the response function. Since $A_{\nu^{\prime} \mathbf{p}^{\prime} ; \nu \mathbf{p}}(a, \mathbf{K})$ appearing when $1 /(a-H)$ acts on e-X pairs, read in terms of both $(S=0)$ and $(S=1)$ trions, the exchange processes, which take place when the two electron spins are identical, have to withdraw the $(S=0)$ trion contributions from the final result. Let us see how they do it.

If we go back to eq. (4.9), we find that the scalar product of e-X states which enters $S_{ \pm, s_{i}}$, has two terms instead of one when the electron spins are the same (see eq. (3.33)). Beside the $\nu_{1}=\nu^{\prime}$ and $\mathbf{p}_{1}=\mathbf{p}_{i}$ term, we also have an exchange term, so that eq. (4.9) now leads to

$$
S_{=}=N_{p} \sum_{\nu^{\prime}, \nu} \lambda_{\nu^{\prime}}\left[A_{\nu^{\prime} \mathbf{p}_{i} ; \nu \mathbf{p}_{i}}\left(a_{i}, \mathbf{K}_{i}\right)-\sum_{\nu_{1}, \mathbf{p}_{1}} L_{\nu^{\prime} \mathbf{p}_{i} ; \nu_{1} \mathbf{p}_{1}} A_{\nu_{1} \mathbf{p}_{1} ; \nu \mathbf{p}_{i}}\left(a_{i}, \mathbf{K}_{i}\right)\right] \lambda_{\nu}^{*}
$$

\subsubsection{Response function in terms of trions}

From eqs. (3.19) and (3.59), we get

$$
\sum_{\nu_{1}, \mathbf{p}_{1}} L_{\nu^{\prime} \mathbf{p}_{i} ; \nu_{1} \mathbf{p}_{1}} A_{\nu_{1} \mathbf{p}_{1} ; \nu \mathbf{p}_{i}}^{(S)}=(-1)^{S} A_{\nu^{\prime} \mathbf{p}_{i} ; \nu \mathbf{p}_{i}}^{(S)}
$$

so that the response function for identical electron spins reads

$$
S_{=}=2 N_{p}|\lambda|^{2} \Omega \sum_{\eta_{1}} \frac{\left|\left\langle\eta_{1} \mid \mathbf{r}=\mathbf{0}, \mathbf{p}_{i}\right\rangle\right|^{2}}{\omega_{p}-\left(E_{\eta_{1}, \mathbf{K}_{i}}^{(T)}-\epsilon_{\mathbf{k}_{i}}^{(e)}\right)+i \eta} .
$$

It thus contains contributions from triplet trions only, as expected. Let us note that, due to this restriction, $S_{=}$cannot be written in a compact form in terms of the trion relative motion Hamiltonian $h_{T}$, as for $S_{\neq}$in eq. (4.23).

\subsubsection{Expansion in e-X diagrams}

We now come back to eq. (4.31) and use the expression of $A_{\nu^{\prime} \mathbf{p}^{\prime} ; \nu \mathbf{p}}(a, \mathbf{K})$ in terms of e-X scaterings given in eq. (3.55). Beside the set of direct ladder diagrams leading to $S_{\neq}$ and which come from the first term of eq. (4.31), there are additional exchange diagrams

coming from the second term. It is of interest to note that these exchanges appear through the $L_{\nu^{\prime}} \mathbf{p}_{i} ; \nu_{1} \mathbf{p}_{1}$ factor which takes place "at the end" of a set of direct e-X scatterings, just 
before the exciton recombination, as $A_{\nu_{1} \mathbf{p}_{1} ; \nu \mathbf{p}_{i}}\left(a_{i}, \mathbf{K}_{i}\right)$ contains direct Coulomb scatterings only. From eq. (4.31) we thus see that, in the case of a photocreated electron having the same spin as the initial electron, the response function in fact reads

$$
S_{=}=S_{\neq}-\hat{S}
$$

where the additional $\hat{S}$ term, coming from possible exchange processes, is given by

$$
\hat{S}=N_{p} \sum_{\nu, \nu_{1}, \mathbf{p}_{1}} \hat{\lambda}_{\mathbf{p}_{i} ; \nu_{1}, \mathbf{p}_{1}} A_{\nu_{1} \mathbf{p}_{1} ; \nu \mathbf{p}_{i}}\left(a_{i}, \mathbf{K}_{i}\right) \lambda_{\nu}^{*}
$$

$\hat{\lambda}_{\mathbf{p} ; \nu_{1}, \mathbf{p}_{1}}$ appears as the exciton-photon vertex $\lambda$ of the bare semiconductor-photon coupling, renormalized by the possible exchanges between the electron of the photocreated exciton and an electron $\mathbf{k}_{i}$ already present in the sample (see fig. (9)). It is precisely given by

$$
\hat{\lambda}_{\mathbf{p} ; \nu_{1}, \mathbf{p}_{1}}=\sum_{\nu} \lambda_{\nu} L_{\nu \mathbf{p} ; \nu_{1} \mathbf{p}_{1}}=\lambda\left\langle\mathbf{p}+\alpha_{e} \mathbf{p}_{1} \mid \nu_{1}\right\rangle
$$

The zero order term of $\hat{S}$ in e-X Coulomb processes comes from the zero order term of $A_{\nu_{1} \mathbf{p}_{1} ; \nu \mathbf{p}_{i}}\left(a_{i}, \mathbf{K}_{i}\right)$ in $C_{\nu^{\prime} \mathbf{p}^{\prime} ; \nu \mathbf{p}}^{\text {dir }}$. Using eq. (3.55), it reads

$$
\hat{S}^{(0)}=N_{p} \sum_{\nu} \frac{\hat{\lambda}_{\mathbf{p}_{i} ; \nu, \mathbf{p}_{i}} \lambda_{\nu}^{*}}{a_{i}-E_{\nu \mathbf{p}_{i} \mathbf{K}_{i}}}=N_{p} \sum_{\nu} \hat{\lambda}_{\mathbf{p}_{i} ; \nu, \mathbf{p}_{i}} G_{X}\left(\omega_{p}, \mathbf{Q}_{p} ; \nu\right) \lambda_{\nu}^{*} .
$$

$\hat{S}^{(0)}$ corresponds to the diagram of fig. (10a), since we have

$$
\hat{\lambda}_{\mathbf{p}_{i} ; \nu, \mathbf{p}_{i}}=-\sum_{\mathbf{k}} \int \frac{i d \omega}{2 \pi} \hat{\lambda}_{\beta_{X} \mathbf{k}-\beta_{e} \mathbf{Q}_{p} ; \nu, \beta_{X} \mathbf{k}-\beta_{e} \mathbf{Q}_{p}} g_{i}^{(e)}(\omega, \mathbf{k})
$$

$\hat{S}^{(0)}$ can also be written in a compact form as

$$
\hat{S}^{(0)}=N_{p}|\lambda|^{2} \sqrt{\Omega}\left\langle\left(1+\alpha_{e}\right) \mathbf{p}_{i}\left|\frac{1}{\omega_{p}-\left(h_{X}+\mathcal{E}_{\mathbf{Q}_{p}}^{(X)}\right)+i \eta}\right| \mathbf{r}=\mathbf{0}\right\rangle .
$$

If we now turn to the first order term, it reads

$$
\hat{S}^{(1)}=\sum_{\nu}\left[\sum_{\nu_{1}, \mathbf{q}_{1}} \frac{\hat{\lambda}_{\mathbf{p}_{i} ; \nu_{1}, \mathbf{p}_{i}-\mathbf{q}_{1}} W_{\nu_{1} \nu}\left(\mathbf{q}_{1}\right)}{\Delta_{\nu_{1}, \mathbf{q}_{1}}}\right] G^{(X)}\left(\omega_{p}, \mathbf{Q}_{p} ; \nu\right) \lambda_{\nu}^{*} .
$$

It is of importance to note that, when the photocreated electron has a spin different from the initial one, the first order term of the response function $S_{\neq}$is zero: The excitonphoton vertex then imposes $\mathbf{p}=\mathbf{p}_{i}$ while $C_{\nu^{\prime} \mathbf{p}^{\prime} ; \nu \mathbf{p}}^{\text {dir }}=0$ for $\mathbf{p}^{\prime}=\mathbf{p}_{i}=\mathbf{p}$. On the opposite, first order e-X scatterings can exist when the two spins are identical, because the possible 
electron exchanges appearing in the dressed exciton-photon vertex do not impose $\mathbf{p}^{\prime}=\mathbf{p}_{i}$ anymore. The first order term of $\hat{S}^{(1)}$ in fact corresponds to the diagram of fig. (10b), since it is easy to show that, for $\mathbf{q}_{1} \neq \mathbf{0}$,

$$
\begin{aligned}
\frac{\hat{\lambda}_{\mathbf{p}_{i} ; \nu_{1}, \mathbf{p}_{i}-\mathbf{q}_{1}}}{\Delta_{\nu_{1}, \mathbf{q}_{1}}} & =-\sum_{\mathbf{k}} \int \frac{i d \omega}{2 \pi} \int \frac{i d \omega_{1}}{2 \pi} \hat{\lambda}_{\beta_{X} \mathbf{k}-\beta_{e} \mathbf{Q}_{p} ; \nu_{1}, \beta_{X} \mathbf{k}-\beta_{e} \mathbf{Q}_{p}-\mathbf{q}_{1}} \\
& \times g_{i}^{(e)}(\omega, \mathbf{k}) g_{i}^{(e)}\left(\omega-\omega_{1}, \mathbf{k}-\mathbf{q}_{1}\right) G_{X}\left(\omega_{p}+\omega_{1}, \mathbf{Q}_{p}+\mathbf{q}_{1} ; \nu_{1}\right) .
\end{aligned}
$$

This first order term can also be written in a compact form as

$$
\begin{array}{r}
\hat{S}^{(1)}=N_{p}|\lambda|^{2} \sqrt{\Omega} \sum_{\mathbf{q}_{1}}\left\langle\left(1+\alpha_{e}\right) \mathbf{p}_{i}-\alpha_{e} \mathbf{q}_{1}\right| \frac{1}{\omega_{p}-\left(h_{X}+\mathcal{E}_{\mathbf{Q}_{p}+\mathbf{q}_{1}}^{(X)}+\epsilon_{\mathbf{k}_{i}-\mathbf{q}_{1}}^{(e)}-\epsilon_{\mathbf{k}_{i}}^{(e)}\right)+i \eta} \\
\times w_{-\mathbf{q}_{1}}(\mathbf{r}) \frac{1}{\omega_{p}-\left(h_{X}+\mathcal{E}_{\mathbf{Q}_{p}}^{(X)}\right)+i \eta}|\mathbf{r}=\mathbf{0}\rangle .
\end{array}
$$

Using the same procedure, we can show that the second order terms of $\hat{S}$ in e-X Coulomb processes correspond to the diagram of fig. (10c) plus the one of fig. (10d). Indeed, here again, the e-X scatterings appearing in $A_{\nu^{\prime} \mathbf{p}_{i} ; \nu \mathbf{p}_{i}}\left(a_{i}, \mathbf{K}_{i}\right)$ impose $\mathbf{q}_{n}-\mathbf{q}_{n-1} \neq \mathbf{0}$ (with $\left.\mathbf{q}_{0} \equiv 0\right)$ : This leads to $\mathbf{q}_{1} \neq \mathbf{0} \neq \mathbf{q}_{2}-\mathbf{q}_{1}$, so that we can have both, $\mathbf{q}_{2} \neq 0$ and $\mathbf{q}_{2}=0$. Since the standard rules for calculating diagrams only lead to processes in which the carriers of the Fermi sea are excited, the diagram of fig. (10c) takes into account $\mathbf{q}_{2} \neq 0$ excitations only. The $\mathbf{q}_{2}=0$ ones which would be missing, have to be included separately through the diagram of fig. (10d).

If we now turn to the third order terms of $\hat{S}$, they correspond to the diagrams of figs. (10e,10f,10g). And so on...

This thus shows that the additional exchange diagrams which take place when the photocreated electron and the initial electron have the same spin, and which are of crucial importance to withdraw the singlet contributions appearing in $S_{\neq}$, correspond to a set of "open" ladder diagrams which have the following characteristics:

(i) They all have one conduction-hole line only, going from left to right, without any hole scattering since the initial "Fermi sea" still contains one electron only.

(ii) They all have an exciton possibly scattered by direct e-X processes without any exchange with the other electron, although the two electrons have the same spin.

(iii) The fact that the exciton can be made with the initial electron or the photocreated electron appears in this case, once and for all, in the renormalized exciton-photon 
interaction $\hat{\lambda}_{\mathbf{p}_{i} ; \nu, \mathbf{p}}$, because the photocreated deep hole can now recombine with any of the two electrons. These diagrams thus start with a bare exciton-photon vertex $\lambda_{\nu}^{*}$ and end with a dressed one $\hat{\lambda}_{\mathbf{p}_{i} ; \nu, \mathbf{p}}$.

The reader can be puzzled by the apparent dissymetry of this new set of diagrams, with the exchange at the end, and not at the beginning. It is actually possible to show that the result does not depend on the position of this exchange.

(iv) Since the scattered exciton can be in a $\left(\nu_{n}, \mathbf{Q}_{p}\right)$ state, while the standard rules for diagrams lead to processes in which the initial "Fermi sea" - here the initial electron - is excited, we must add to the connected "open" ladder diagrams, diagrams with an "open" part separated from a ladder part, as in fig. (10d,10f,10g).

\subsection{Volume dependence}

From the expressions of the response functions $S_{\neq}$and $S_{=}$in terms of trions given in eqs. (4.22) and (4.33), we see that the trion oscillator strength reads simply in terms of the Fourier transform of the trion relative motion wave function $\left\langle\mathbf{r}, \mathbf{u} \mid \eta_{S}\right\rangle$, written with the "good" trion variables $\mathbf{r}$ and $\mathbf{u}$, defined in eqs. (3.2) and (3.10). We also see that, when the photon polarization is such that only $(S=1)$ trions can be formed, the oscillator strength of these $(S=1)$ trions have an additional factor of 2 , compared to the case in which both $(S=0)$ and $(S=1)$ trions can be created.

From the expression $|\lambda|^{2} \Omega\left|\left\langle\mathbf{r}=\mathbf{0}, \mathbf{p}_{i} \mid \eta\right\rangle\right|^{2}$ of the trion oscillator strength obtained in eq. (4.22), it is easy [19] to compare its size for bound state (eeh), partially dissociated state $(e+e h)$ and totally dissociated state $(e+e+h)$.

In the case of a bound trion, $\langle\mathbf{r}, \mathbf{u} \mid \eta\rangle$ has an extension over $r$ of the order of $a_{X}$ and an extension over $u$ of the order of $a_{T}$, with $a_{T}$ much larger than $a_{X}$ since the trion binding energy is much weaker than the exciton binding energy. In 2D, dimensional arguments then lead to $\langle\mathbf{r}=\mathbf{0}, \mathbf{u}=\mathbf{0} \mid \eta\rangle \sim\left(a_{X} a_{T}\right)^{-1}$. From the spatial extension $a_{T}$ of $\langle\mathbf{r}=\mathbf{0}, \mathbf{u} \mid \eta\rangle$, we then find, again from dimensional arguments, that, for $p \ll a_{T}^{-1}$, $\langle\mathbf{r}=\mathbf{0}, \mathbf{p} \mid \eta\rangle \sim\langle\mathbf{r}=\mathbf{0}, \mathbf{u}=\mathbf{0} \mid \eta\rangle\langle\mathbf{p} \mid \mathbf{u} \simeq \mathbf{0}\rangle a_{T}^{2} \sim a_{T} / a_{X} L$, where $L$ is the sample size $\left(L^{2}=\Omega\right)$. This leads to a bound trion oscillator strength $\sim|\lambda|^{2}\left(a_{T} / a_{X}\right)^{2}$, independent from the sample volume.

If we now consider a partially dissociated trion, $\langle\mathbf{r}, \mathbf{u} \mid \eta\rangle$ now has an extension $a_{X}$ 
over $r$ but $L$ over $u$, so that $\langle\mathbf{r}=\mathbf{0}, \mathbf{u}=\mathbf{0} \mid \eta\rangle \sim\left(a_{X} L\right)^{-1}$. For $p$ small, we then have $\langle\mathbf{r}=\mathbf{0}, \mathbf{p} \mid \eta\rangle \sim 1 / a_{X}$, so that the partially disssociated trions have an oscillator strength $\sim|\lambda|^{2} L^{2} / a_{X}^{2}$, which is the one of a free exciton.

Finally, for a totally dissociated trion, $\langle\mathbf{r}, \mathbf{u} \mid \eta\rangle$ has an extension $L$ over $r$ and $L$ over $u$, so that $\langle\mathbf{r}=\mathbf{0}, \mathbf{u}=\mathbf{0} \mid \eta\rangle \sim L^{-2}$. For small $p$, this gives $\langle\mathbf{r}=\mathbf{0}, \mathbf{p} \mid \eta\rangle \sim 1 / L$, which leads to a sample volume independent oscillator strength $|\lambda|^{2}$ equal to the one of the exciton diffusive states, $i$. e., free carriers. This oscillator strength is however smaller than the bound trion one, since the trion extension $a_{T}$ is somewhat larger than the exciton extension $a_{X}$

We thus conclude that the partially dissociated trion has essentially the same oscillator strength as the exciton, while the bound trion oscillator strength is $a_{T}^{2} / L^{2}$ smaller. It is thus vanishingly small in the large sample limit, $i$. e., the limit of solid state physics. This is after all not surprising: If we take a large sample and if we add just one electron, we cannot expect any sizeable change in the photon absorption spectrum! From a technical point of view, if we consider the volume dependence of the diagrams representing the response function, we find that the dominant one corresponds to the bare exciton diagram, given in fig. (7a), so that in the large $L$ limit, the trion diagrams reduce to this unique diagram.

These quite simple arguments lead us to conclude that, when a line is seen, well below the exciton line, in the photon absorption spectrum of a doped semiconductor, it cannot be the line of a (clean) trion because its oscillator strength would be a sample volume (or a coherence volume) smaller than the exciton one. It is most probably a trion having many-body effects with the $(N-1)$ other electrons of the semiconductor, or better a photocreated exciton dressed by the $N$ electrons already present in the sample. This quite difficult many-body problem will be adressed in a further work, using the tools we have established in sections 3 and 4 of the present paper, which allow to treat excitons interacting with electrons through electron-exciton diagrams. 


\section{Conclusion}

In order to solve the simplest problem on trion, namely the photon absorption in the presence of one electron, we have first shown that the standard Feynman diagrams, which read in terms of free electrons and free holes, are totally inappropriate: As the trion is a bound state, Coulomb processes have to be included at all orders. While these Coulomb processes can be handled exactly between the two carriers of an exciton, it is totally hopeless to draw and sum them all, in the case of three carriers.

We propose to reduce this three-body problem to a two-body problem by considering the trion as an electron interacting with an exciton. Although physically appealing, this approach however faces the problem of the electron indistinguishability, $i$. e., the fact that the exciton can a priori be made with any of the two electrons. Our commutation technique, designed to deal with this problem in an exact way, allows to overcome this difficulty.

We find that when the photocreated electron has a spin different from the initial electron spin - which is for example what happens if we want to photocreate a ground state trion - , we can forget about these possible electron exchanges: The response function to the photon field just corresponds to a set of e-X ladder diagrams between e-X pairs $(\nu, \mathbf{p}, \mathbf{K})$, with $\mathbf{K}$ being the center of mass momentum of the pair — constant in these ladder processes - , $\mathbf{p}$ the relative motion momentum of the e-X pair and $\nu$ characterizing the exciton relative motion level. The e-X interaction vertex $C_{\nu^{\prime} \mathbf{p}^{\prime} ; \nu \mathbf{p}}^{\mathrm{di}}$ of these novel diagrams corresponds to direct Coulomb processes between the electron and the exciton, the "in" exciton $\nu$ and the "out" exciton $\nu^{\prime}$ being made with the same electron. $C_{\nu^{\prime} \mathbf{p}^{\prime} ; \nu \mathbf{p}}^{\text {dir }}$ reads in terms of the Fourier transform $w_{\mathbf{q}}(\mathbf{r})$ of the Coulomb potential $w(\mathbf{r}, \mathbf{u})$ between the electron $e^{\prime}$ and the exciton made of $(e, h)$.

The possible electron exchanges are only important when the spins of the two electrons are identical, $i$. e., when only triplet trions $\left(S_{z}= \pm 1\right)$ are photocreated. It is then possible to include these exchanges through a dressed exciton-photon interaction. They thus play a role, once and for all, when the hole recombines, so that we are again left with direct e-X ladder processes only, except that they are now "open".

The physical reason for a so trivial consequence of the possible electron exchange lies in the fact that two exchanges reduce to an identity; so that, either we end with no exchange 
at all - as when the photocreated hole can recombine with the photocreated electron only, which is what happens when the two electron spins are different - , or we end with zero and one exchange - as when the hole can recombine with either the photocreated electron or the initial electron, which is what happens when the two electrons have the same spin.

In section 3 of this paper, we have also collected all important results on trions derived in our previous works — plus some unpublished ones. This "background on trion", which leads to this novel many-body procedure in terms of electrons and excitons, will be of great help to study the interaction of trion with carriers in doped semiconductors: Indeed, the existing literature on this very difficult many-body problem, which relies on standard electron-hole procedure - the only one known up to now —, is quite unsatisfactory, as we outlined in ref. [19].

\section{REFERENCES}

(1) E. Hylleraas, Phys. Rev. 75, 491 (1947).

(2) M. Lampert, Phys. Rev. Lett. 1, 450 (1958).

(3) K. Kheng, R.T. Cox, Y. Merle d'Aubigné, F. Bassani, K. Saminadayar, S. Tatarenko, Phys. Rev. Lett. 71, 1752 (1993).

(4) G. Finkelstein, H. Shtrikman, I. Bar Joseph, Phys. Rev. Lett. 74, 976 (1995).

(5) S.A. Brown, J.F. Young, J.A. Brum, P. Hawrylak, Z. Wailewski, Phys. Rev. B 54, R11082 (1996).

(6) G. Astakhov, V.P. Kochereshko, D.R. Yakovley, W. Ossau, J. Nurnberger, W. Faschinger, G. Landwehr, Phys. Rev. B 62, 10345 (2000).

(7) P. Redlinski, J. Kossut, Solid State Com. 118, 295 (2001).

(8) J. Puls, G.V. Mikhailov, F. Henneberger, D.R. Yakovley, A. Waag, W. Faschinger, Phys. Rev. Lett. 89, 287402 (2002).

(9) D.V. Kulakovskii, Y.E. Lozovik, J.E.T.P. Lett. 76, 516 (2002).

(10) B. Stebe, G. Munschy, Solid State Com. 17, 1051 (1975).

(11) P. Hawrylak, Phys. Rev. B 44, 3821 (1991). 
(12) B. Stébé, G. Munschy, L. Stauffer, F. Dujardin, J. Murat, Phys. Rev. B 56, 12454 (1997).

(13) B. Stébé, E. Feddi, A. Ainane, F. Dujardin, Phys. Rev. B 58, 9926 (1998).

(14) A. Esser, E. Runge, R. Zimmerman, W. Langbein, Phys. Rev. B 62, 8232 (2000).

(15) W. Florek, J. Math. Phys. 42, 5177 (2001).

(16) L.C. Dacal, R. Ferreira, G. Bastard, J.A. Brum, Phys. Rev. B 65, 115325 (2002).

(17) M. Combescot, Eur. Phys. J. B 33, 311 (2003).

(18) M. Combescot, O. Betbeder-Matibet, Solid State Com. 126, 687 (2003).

(19) M. Combescot, J. Tribollet, Solid State Com. 128, 273 (2003).

(20) M. Combescot, P. Nozières, J. de Phys. (Paris), 32, 913 (1971).

(21) We draw diagrams with arrows from right to left, while one usually draws them from left to right. Since diagrams basically represent matrix elements, our way makes these diagrams to topologically appear just as the algebraic expressions they represent. This is why we find this drawing more convenient to read than the one found in usual textbooks on many-body effects.

(22) M. Combescot, O. Betbeder-Matibet, Europhys. Lett. 58, 87 (2002).

(23) O. Betbeder-Matibet, M. Combescot, Eur. Phys. J. B, 27, 505 (2002).

(24) M. Combescot, O. Betbeder-Matibet, Europhys. Lett. 59, 579 (2002).

(25) M. Combescot, O. Betbeder-Matibet, Cond-mat/0402087, submitted to Phys. Rev. Lett..

(26) M. Combescot, O. Betbeder-Matibet, K. Cho, H. Ajiki, Cond-mat/0311387, submitted to Phys. Rev. Lett. 


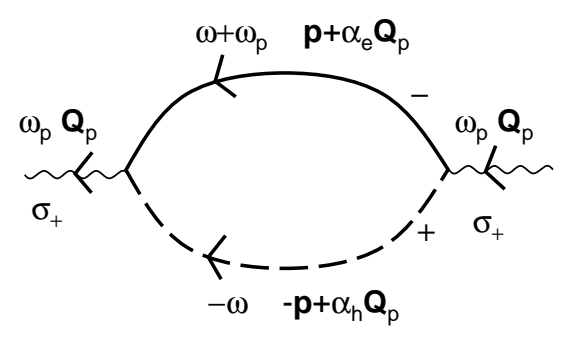

(a)

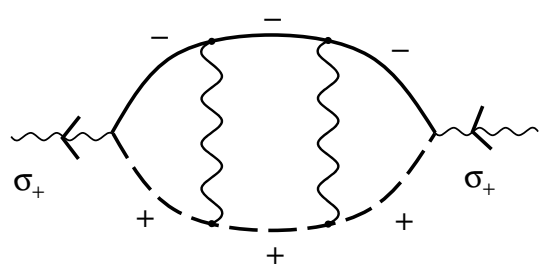

(c)

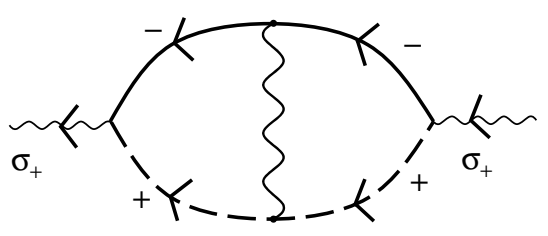

(b)

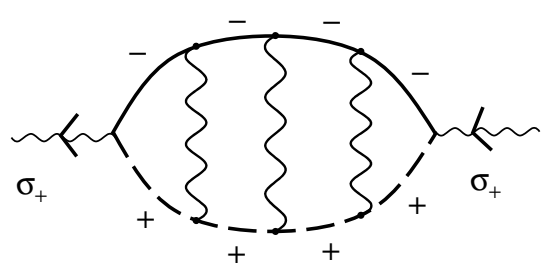

(d)

Figure 1: Photon absorption with exciton formation: well-known set of electron-hole ladder diagrams with 0, 1, 2, 3...electron-hole Coulomb interactions, (a), (b), (c), (d) respectively. Solid line: electron. Dashed line: hole. Wavy line: Coulomb interaction between electron and hole. In a quantum well, a $\sigma_{+}$photon creates a hole with momentum $(+3 / 2)$, noted + , and an electron with spin $(-1 / 2)$, noted - . 

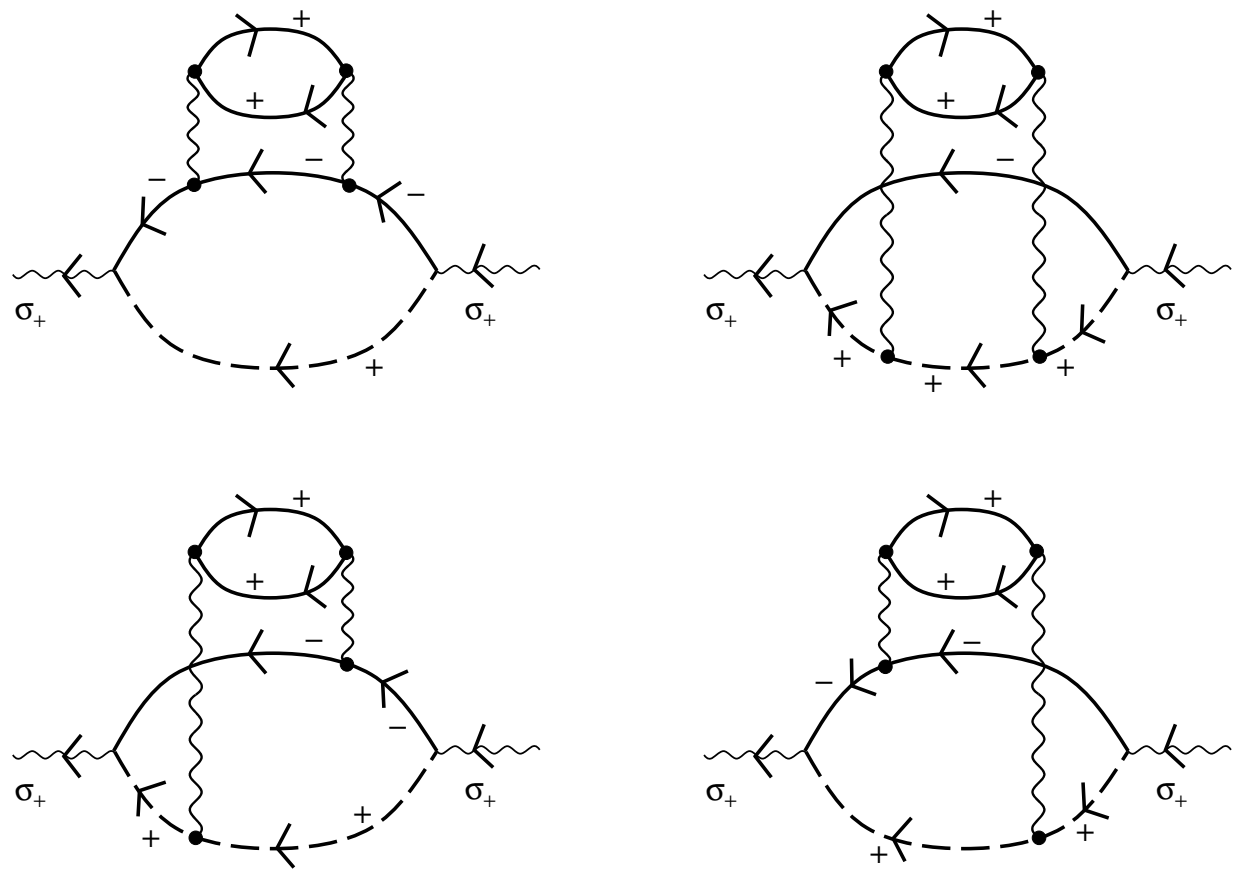

Figure 2: Absorption of a $\sigma_{+}$photon with trion formation, in the case of a photocreated electron and an initial electron having different spins: second order in Coulomb interaction. The diagrams of this figure have to be added to the one of fig. (1c). 

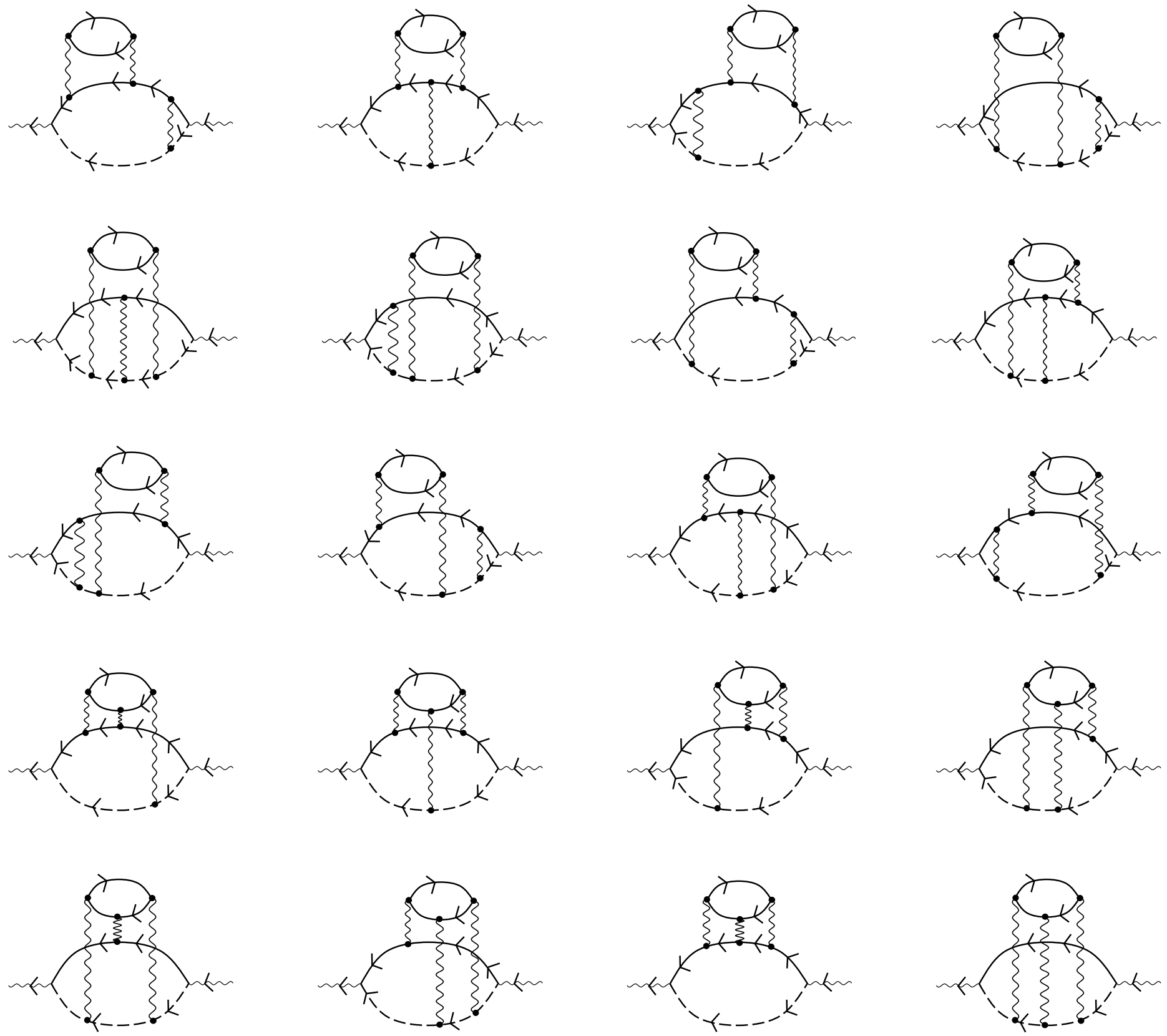

Figure 3: Same as fig. (2), with three Coulomb interactions. The diagrams of this figure have to be added to the one of fig. (1d). 


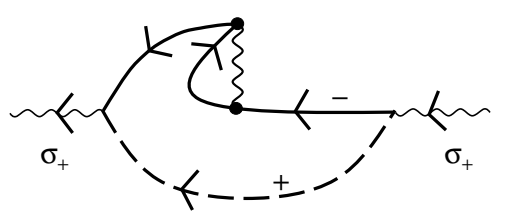

(a)
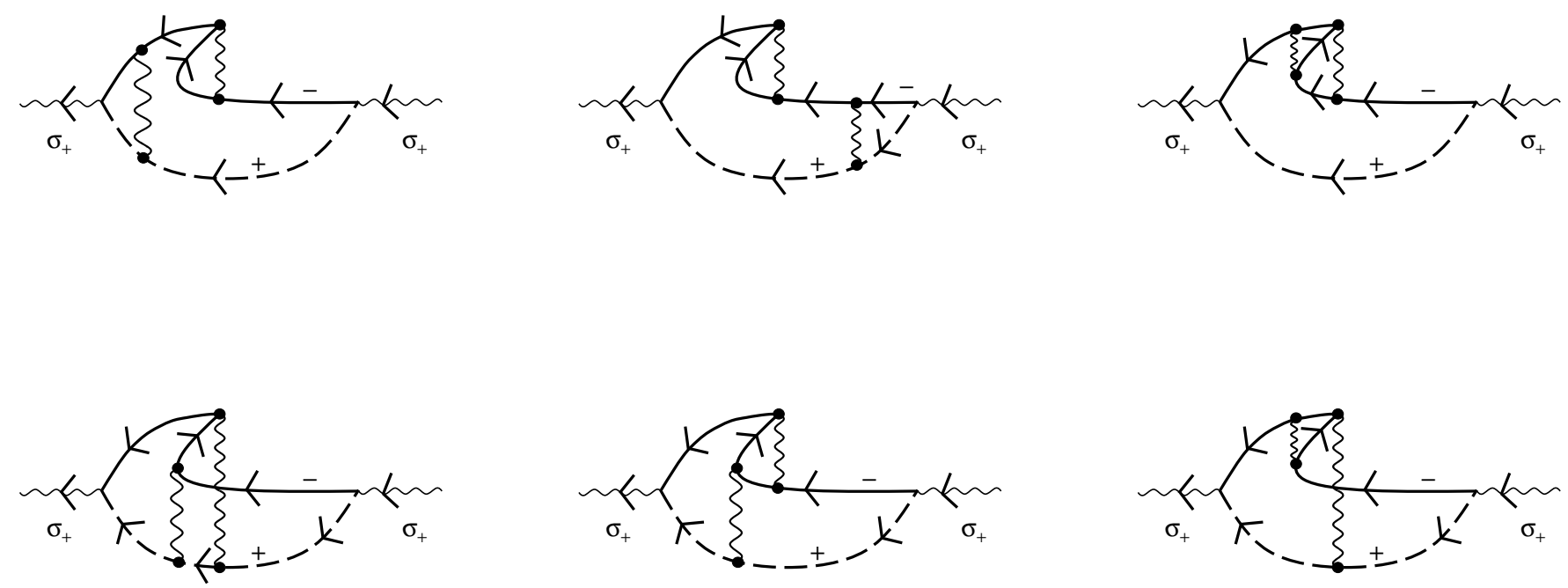

(b)

Figure 4: Absorption of a $\sigma_{+}$photon with trion formation, in the case of a photocreated electron and an nitial electron having the same spin, at first order (a) and second order (b) in Coulomb interaction. The diagrams of this figure, which correspond to possible exchange between the photocreated electron and the initial electron, have to be added to the diagram of fig. (1b) and the diagram of fig. (1c) plus the ones of fig. (2), respectively. 


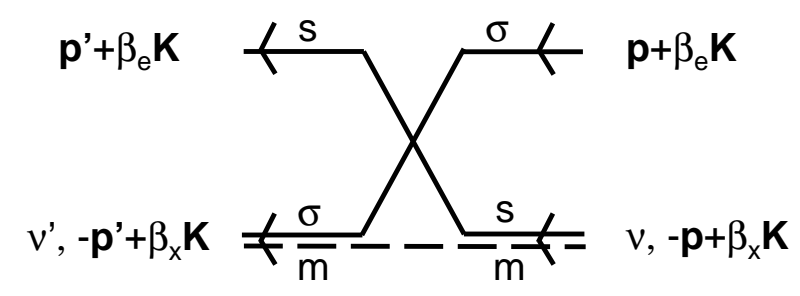

(a)

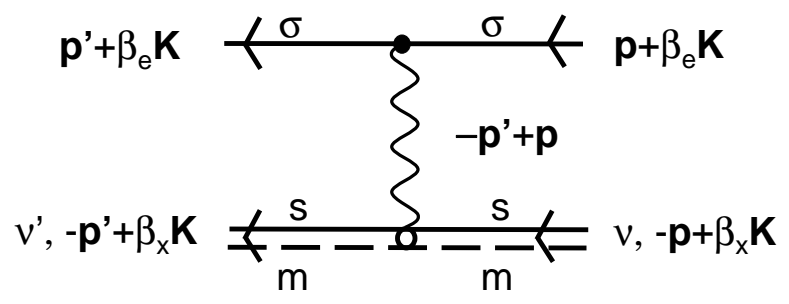

(b)

Figure 5: (a) Exchange parameter $L_{\nu^{\prime} \mathbf{p}^{\prime} ; \nu \mathbf{p}}$ of the "commutation technique". The "in" exciton $\nu$ and the "out" exciton $\nu^{\prime}$ are made with different electrons. No Coulomb interaction takes place in this scattering. (b) Direct coulomb scattering $C_{\nu^{\prime} \mathbf{p}^{\prime} ; \nu \mathbf{p}}^{\mathrm{dir}}$ of the "commutation technique". The "in" exciton $\nu$ and the "out" exciton $\nu^{\prime}$ are made with the same electron. 

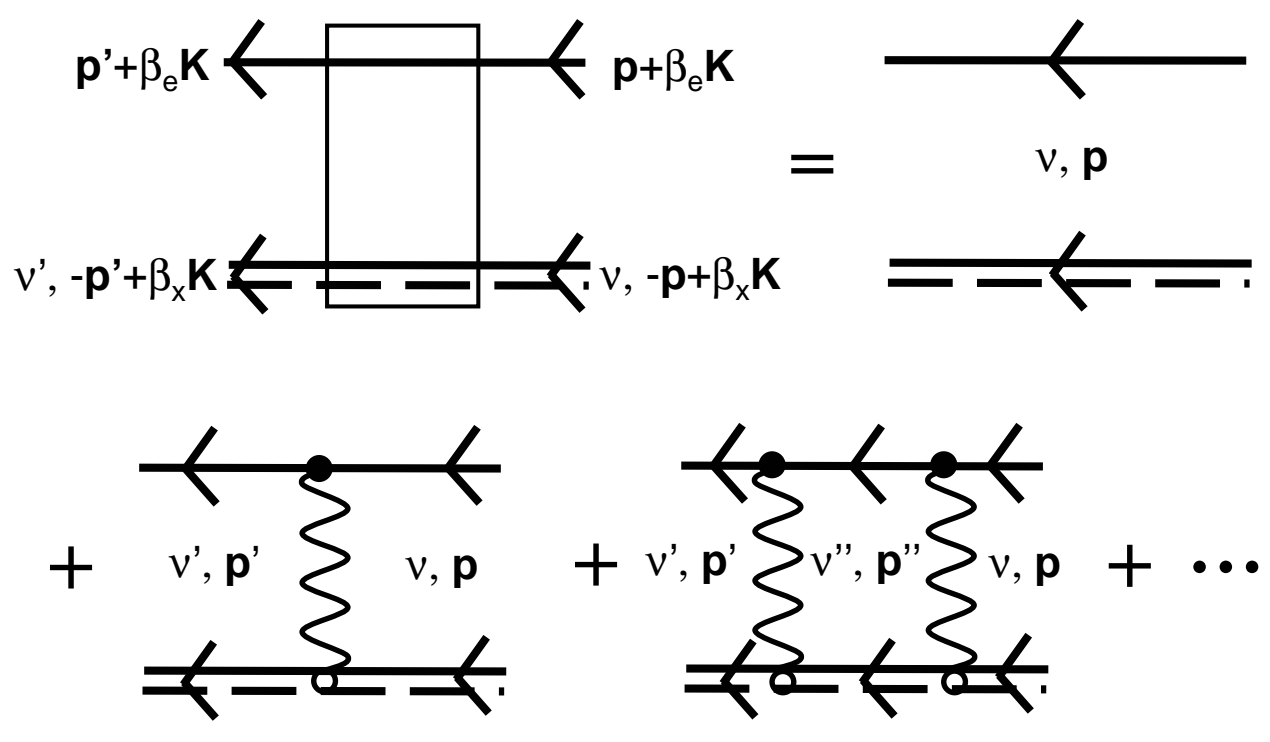

Figure 6: Set of ladder processes between one electron and one exciton. Note that, all over, the exciton is made with the same electron and the e-X pair has the same center of mass momentum $\mathbf{K}$. The exciton quantum number, $\nu$, and the pair relative motion momentum, p, are the only things which change in these ladder processes. The solid line corresponds to the electron. The double solid-dashed line corresponds to the exciton. The wavy line corresponds to the direct Coulomb scattering $C_{\nu^{\prime} \mathbf{p}^{\prime} ; \nu \mathbf{p}}^{\mathrm{dir}}$. 


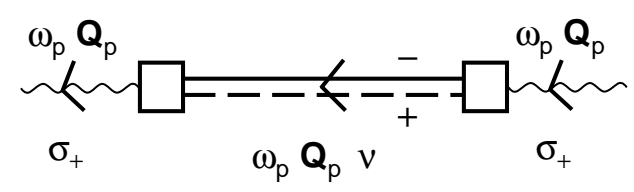

(a)

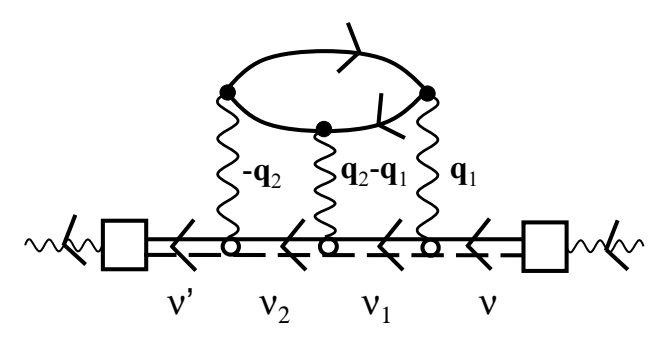

(c)

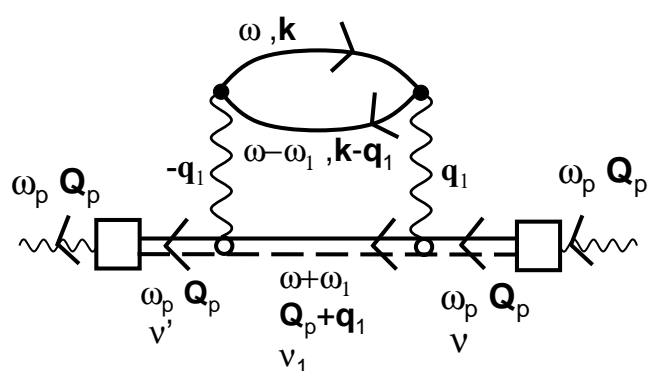

(b)

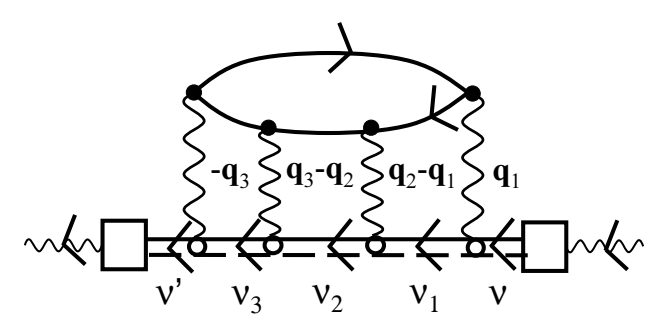

(d)

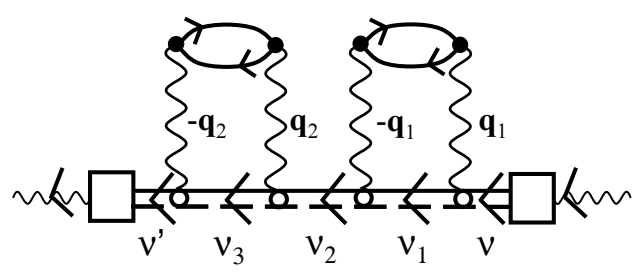

(e)

Figure 7: (a) Absorption of a $\sigma_{+}$photon with exciton formation, using exciton diagrams. This unique diagram corresponds to the set of electron-hole ladder diagrams of fig. (1). It also corresponds to the trion absorption diagram, at zero order in Coulomb scattering $C_{\nu^{\prime} \mathbf{p}^{\prime} ; \nu \mathbf{p}}^{\mathrm{dir}}$. (b) Absorption of a $\sigma_{+}$photon with trion formation, when the photocreated electron and the initial electron have different spins: second order process in (direct) Coulomb scattering between electron and exciton $C_{\nu^{\prime} \mathbf{p}^{\prime} ; \nu \mathbf{p}}^{\mathrm{dir}}$. (c) Same as (b), with three direct Coulomb scatterings. (d) Same as (b), with four direct Coulomb scatterings. (e) Additional diagram which appears in the response function at fourth order in $C_{\nu^{\prime} \mathbf{p}^{\prime} ; \nu \mathbf{p}}^{\mathrm{dir}}$. 


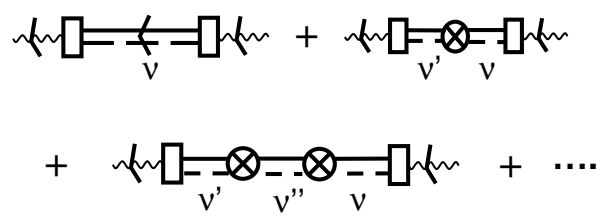

(a)

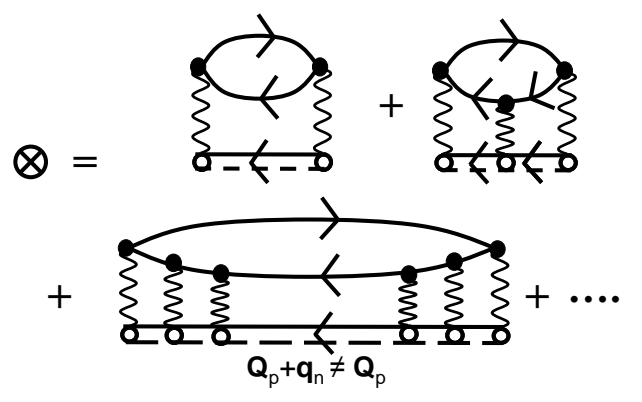

(b)

Figure 8: (a) Integral equation (4.28) for the response function $S_{\neq}$when the photocreated electron and the initial electron have different spins. The cross corresponds to all possible diagrams shown in fig. (8b). (b) Processes contributing to the exciton scattering $\Gamma_{\nu^{\prime} \nu}$ appearing in the integral equation (4.28): In these "bubbles", the exciton momentum always differs from the initial momentum $\mathbf{Q}_{p}$, by construction.

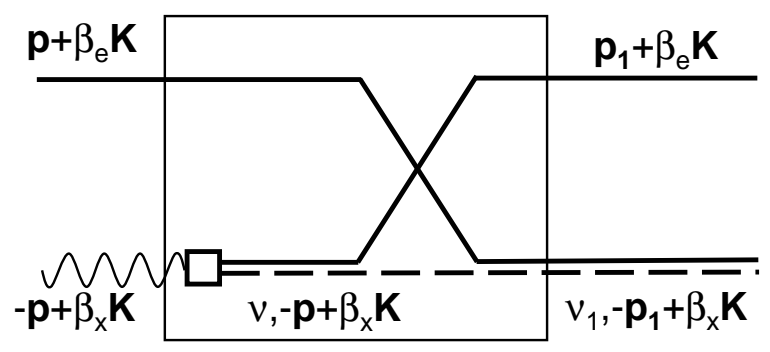

Figure 9: Semiconductor-photon interaction $\hat{\lambda}_{\mathbf{p} ; \nu_{1} \mathbf{p}_{1}}$ dressed by the presence of one electron $\mathbf{k}=\mathbf{p}+\beta_{X} \mathbf{K}$ having the same spin as the photocreated electron. 


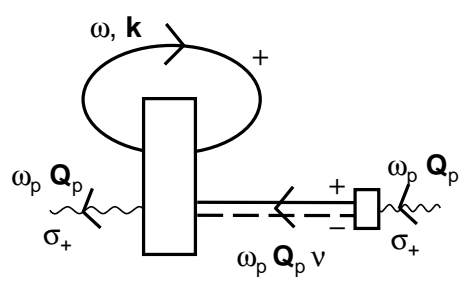

(a)

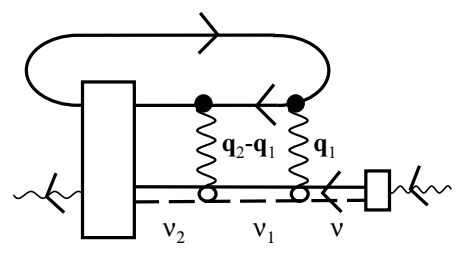

(c)

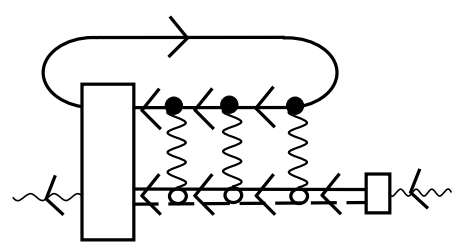

(e)

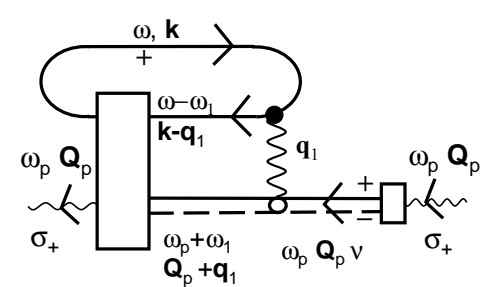

(b)

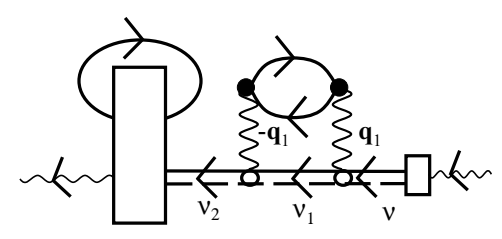

(d)

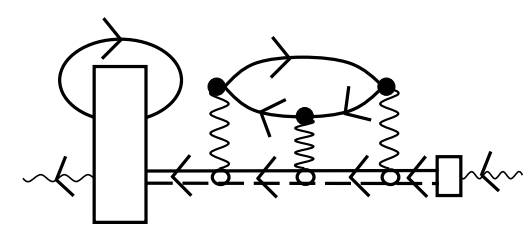

(f)

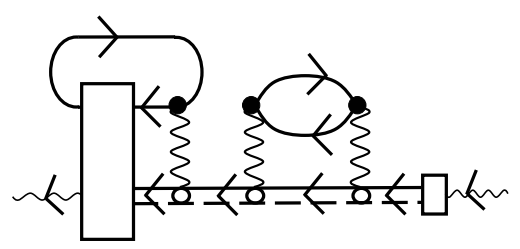

(g)

Figure 10: Photon absorption with trion formation when the photocreated electron and the initial electron have the same spin. The diagrams of this figure have to be added to the diagrams of fig. (7). Their main effect is to withdraw the $S=0$ trion contributions which cannot exist when the two electron spins are identical. (a) Zero order in direct Coulomb electron-exciton scattering: The photocreated exciton already feels the presence of the initial electron through exchange processes included in the dressed semiconductor-photon interaction. (b) First order in $C_{\nu_{1} \mathbf{p}_{1} ; \nu \mathbf{p}}^{\text {dir }}$ (c) and (d) Second order. (e), (f) and (g) Third order. 